

\title{
Explicit algebraic Reynolds stresses and scalar fluxes for density-stratified shear flows
}

D Violeau

\section{To cite this version:}

D Violeau. Explicit algebraic Reynolds stresses and scalar fluxes for density-stratified shear flows. Physics of Fluids, 2009, 21, pp.35103. 10.1063/1.3081552 . hal-01097837

\section{HAL Id: hal-01097837 \\ https://hal.science/hal-01097837}

Submitted on 22 Dec 2014

HAL is a multi-disciplinary open access archive for the deposit and dissemination of scientific research documents, whether they are published or not. The documents may come from teaching and research institutions in France or abroad, or from public or private research centers.
L'archive ouverte pluridisciplinaire HAL, est destinée au dépôt et à la diffusion de documents scientifiques de niveau recherche, publiés ou non, émanant des établissements d'enseignement et de recherche français ou étrangers, des laboratoires publics ou privés. 


\title{
Explicit algebraic Reynolds stresses and scalar fluxes for density-stratified shear flows
}

\author{
D. Violeau
}

Saint-Venant Laboratory for Hydraulics - Université Paris-Est, joint research unit EDF R\&D - CETMEF - Ecole des Ponts, 6 quai Watier, 78400 Chatou, France - damien.violeau@edf.fr

\section{Abstract}

An Explicit Algebraic Model for Reynolds stresses and active scalar turbulent fluxes is proposed for simply-stratified, stable shear flows like atmospheric boundary layers of estuaries, including buoyant effects due to density variations (temperature, salinity). Under equilibrium assumptions, this models leads to explicit functions of the gradient Richardson number $R i$ that model the damping of the eddy viscosity and eddy diffusivity coefficients. The present model is compared to other existing theoretical approaches, as well as experimental observations, with satisfactory agreement. The evolution of the turbulent Prandtl number as a function of $R i$ is correctly predicted and its neutral value is consistent with existing values in the literature. The behaviour of the model predicts that internal waves are responsible for momentum diffusion at large $R i$, consitently with recent publications. The properties of turbulence anisotropy are briefly investigated.

\section{Introduction}

The question of modelling the turbulent fluxes of momentum together with active scalars is at the heart of engineering and computational fluid dynamics. In particular, many attempts have been made to modify the traditional eddyviscosity and eddy-diffusivity models, i.e. to derive damping functions allowing the correct estimation of diffusion parameters under stable density stratification in the atmopsheric boundary layer, rivers and estuaries. Since the measurements by Munk and Anderson, ${ }^{1}$ the most consistent developments have been achieved by Mellor and Yamada ${ }^{2}$ on theoretical backgrounds. However, this model and similar ones present some drawbacks; in particular they predict a critical value of the Richardson number above which turbulence is destroyed by buoyancy, while the most recents observations and simulations ${ }^{3}$ lead to the conclusion that turbulent diffusion survives at high Richardson numbers through the mechanism of internal waves. On the other hand, since Pope, ${ }^{4}$ Explicit Algebraic Reynolds 
Stress Models (EARSM) have improved the theoretical basis of our knowledge on turbulent momentum fluxes, while several authors have extended this approach to scalar fluxes ${ }^{5}$. These models, however, often suffer from a lack of consistency in the modelling of buoyant terms. We propose here a more rigorous approach, based on a relevant decomposition of flux components on appropriate basis.

In the first section, the governing equations of the considered closure models for Reynolds stresses and scalar fluxes with buoyancy forces are presented. In the second section, we focus on 2-D flows, i.e. invariant along a (spanwise) direction, and we derive a subsequent linear system of five equations and five unknowns to estimate the turbulent fluxes; an exact solution is given for passive scalars. In the particular case of simple, stably stratified shear flows, we also give an exact solution and show how the latter turns into a set of analytical damping functions. In the third and last section, the performances of the proposed model are examined from other published models and experimental data. We extend our model to take account of internal waves for highly stratified flows. The properties of our model in terms of anisotropy and realizability are finally briefly investigated.

\section{Basic governing equations}

\subsection{Reynolds stresses}

In this section, we summarize the set of model equations on the basis of which we will establish our model in the subsequent sections; we also introduce our notation. We refer here to well-known models widely mentioned in the literature. We consider the general case of a turbulent flow with temperature, salinity, or any other active scalar, i.e. modifying the fluid density. However, we assume that the temperature (or scalar concentration), hereafter denoted by $T$, is small enough to yield moderate density variation, so that Boussinesq's approximation stands, i.e. the density variations $\delta \rho$ only affect the gravity term, leading to buoyancy forces proportional to $\delta \rho$. For a flow with slighly variable density, the Reynolds-averaged forms of the Navier-Stokes momentum equation and the scalar equation then read

$$
\begin{aligned}
\frac{D \overline{\mathbf{u}}}{D t} & =-\frac{1}{\rho} \nabla \bar{p}+\nu \Delta \overline{\mathbf{u}}-\nabla \cdot \mathbf{R}+\beta_{T} \bar{T} \mathbf{g} \\
\frac{D \bar{T}}{D t} & =K \Delta \bar{T}-\nabla \cdot \mathbf{Q}_{T}
\end{aligned}
$$

where $\mathbf{u}$ and $p$ denote the velocity and the pressure fields while the overbars refer to Reynolds-averaging and the $D / D t$ to a lagrangian derivative (i.e. including advection) following the Reynolds-averaged (or "mean") velocity $\overline{\mathbf{u}}$. Besides, the constants $\rho, \nu, K$ and $\beta_{T}$ are the reference fluid density and molecular kinematic viscosity and the scalar molecular diffusivity and expansion co-

efficient, respectively. The latter is defined from the density variation due to 
the presence of the active scalar by

$$
\beta_{T}=-\frac{\delta \rho}{\rho \bar{T}}
$$

$\beta_{T}$ is thus positive for temperature, negative for salt. Finally, $\mathbf{g}$ is the gravity vector, while the Reynolds stress tensor and the turbulent scalar flux vector are defined by

$$
\begin{aligned}
\mathbf{R} & =\overline{\mathbf{u}^{\prime} \otimes \mathbf{u}^{\prime}} \\
\mathbf{Q}_{T} & =\overline{T^{\prime} \mathbf{u}^{\prime}}
\end{aligned}
$$

where $\mathbf{u}^{\prime}=\mathbf{u}-\overline{\mathbf{u}}$ and $T^{\prime}=T-\bar{T}$ are the turbulent fluctuating velocity and scalar fields (we call these quantities 'fluxes' although this role is assumed by $-\rho \mathbf{R}$ and $-\rho \mathbf{Q}_{T}$, respectively). $\mathbf{R}$ represents a stress tensor, in other terms a turbulent flux of mean linear momentum. Still under the Boussinesq assumption, the Reynolds-averaged continuity equation reads

$$
\nabla \cdot \overline{\mathbf{u}}=0
$$

The above equations would form a closed system if we knew an exact analytical form for $\mathbf{R}$, which is known to be impossible from pure mathematical considerations, due to the non-linearity of Navier-Stokes equations. It is known that any attempt of deriving exact equations for statistical momenta of order $n$ raises to new terms, in particular momenta of immediately higher order. Thus, closure assumptions based on heuristic models are needed to close the system of equations. Such a closure model is the standard Boussinesq eddy-viscosity based model (see section 5.1), which is a first order model in that sense that it closes the system at the level of the second order statistical momenta, namely R. In the following, we focus on a more general approach, referred to as Explicit Algebraic Reynolds Stress Models (EARSM). EARSM are also first order closure models, but they stem from approximate solutions of a second order closure model. Before coming to the construction of a solution (section 4), we now describe the second order model used for this purpose. We first consider the equation governing $\mathbf{R}$, which requires closure assumptions, according to the abovementioned considerations. In a general form, this tensorial equation reads

$$
\frac{D \mathbf{R}}{D t}=\mathbf{P}_{\mathbf{R}}+\mathbf{G}_{\mathbf{R}}+\mathbf{D}_{\mathbf{R}}+\boldsymbol{\Phi}_{\mathbf{R}}-\varepsilon_{\mathbf{R}}
$$

where $\mathbf{P}_{\mathbf{R}}, \mathbf{G}_{\mathbf{R}}, \mathbf{D}_{\mathbf{R}}, \mathbf{\Phi}_{\mathbf{R}}$ and $\varepsilon_{\mathbf{R}}$ are respectively production, buoyancy, diffusion, pressure-strain correlation and dissipation tensors. In this section, we will describe these terms and the models considered here to close equation (5). However, we will not examine the diffusion $\mathbf{D}_{\mathbf{R}}$, for reasons which will appear in section 4.1; this will remain true for all diffusion terms in this work, as we will see below. The first two terms of the right-hand-side of (5) can be written exactly (i.e. from the Navier-Stokes equations, without any closure model). The production is explicitely given by

$$
\mathbf{P}_{\mathbf{R}}=-\mathbf{R} \boldsymbol{\Gamma}_{u}^{T}-\boldsymbol{\Gamma}_{u} \mathbf{R}
$$


where the gradient of the mean velocity field is denoted by

$$
\boldsymbol{\Gamma}_{u}=\nabla \overline{\mathbf{u}}=\frac{\partial \bar{u}_{i}}{\partial x_{j}} \mathbf{e}_{i} \otimes \mathbf{e}_{j}
$$

(here and in the following, Einstein's summation convention on repeated labels occurs). Equation (6) may be rewritten after introducing the following notations. We first define the mean rate-of-strain and vorticity tensors by

$$
\begin{aligned}
\mathbf{S} & =\frac{1}{2}\left(\boldsymbol{\Gamma}_{u}+\boldsymbol{\Gamma}_{u}^{T}\right)=\boldsymbol{\Gamma}_{u}^{S} \\
\boldsymbol{\Omega} & =\frac{1}{2}\left(\boldsymbol{\Gamma}_{u}-\boldsymbol{\Gamma}_{u}^{T}\right)
\end{aligned}
$$

where the superscript ${ }^{S}$ refers to the symetric part of a tensor. Note that (4) can now be rewritten

$$
\operatorname{tr} \mathbf{S}=0
$$

We also define the turbulent kinetic energy by

$$
k=\frac{1}{2} \operatorname{tr} \mathbf{R}
$$

In most of the turbulent closure models, $k$ is given by a governing equation (see later), so that the remaining unknown in the Reynolds stresses is the (dimensionless) anisotropy tensor, defined by

$$
\mathbf{a}=\frac{1}{k} \mathbf{R}^{D}=\frac{1}{k} \mathbf{R}-\frac{2}{3} \mathbf{I}
$$

where the superscript ${ }^{D}$ refers to the deviatoric part of a tensor. With these notations, (6) reads

$$
\mathbf{P}_{\mathbf{R}}=-k\left(\mathbf{a S}+\mathbf{S a}-\mathbf{a} \boldsymbol{\Omega}+\boldsymbol{\Omega} \mathbf{a}+\frac{4}{3} \mathbf{S}\right)
$$

The buoyancy tensor is also an explicit term :

$$
\mathbf{G}_{\mathbf{R}}=-2 \beta_{T}\left(\mathbf{g} \otimes \mathbf{Q}_{T}\right)^{S}
$$

However, like $\mathbf{R}, \mathbf{Q}_{T}$ remains unknown and will require a closure model. Thus the buoyancy tensor is not totally explicit, but will be so given an appropriate model for $\mathbf{Q}_{T}$ (see section 3.2). Coming back to equation (5), contrary to $\mathbf{R}$, the pressure-strain correlation tensor $\boldsymbol{\Phi}_{\mathbf{R}}$ now requires a closure form (its exact analytical expression in terms of turbulent fluctuating components will not be given here ${ }^{6}$. We will consider herein the LRR-QI model: ${ }^{7}$

$$
\mathbf{\Phi}_{\mathbf{R}}=-C_{R R} \frac{\varepsilon}{k} \mathbf{R}^{D}-C_{R P} \mathbf{P}_{\mathbf{R}}^{D}-C_{R Q} \mathbf{Q}^{D}-C_{R G} \mathbf{G}_{\mathbf{R}}^{D}-C_{R S} k \mathbf{S}
$$


In the above equation, $\mathbf{Q}$ is a new tensor defined by

$$
\begin{aligned}
\mathbf{Q} & =-\mathbf{R} \boldsymbol{\Gamma}_{u}-\boldsymbol{\Gamma}_{u}^{T} \mathbf{R} \\
& =-k\left(\mathbf{a} \mathbf{S}+\mathbf{S a}+\mathbf{a} \boldsymbol{\Omega}-\mathbf{\Omega} \mathbf{a}+\frac{4}{3} \mathbf{S}\right)
\end{aligned}
$$

while $C_{R R}, C_{R P}, C_{R Q}, C_{R G}$ and $C_{R S}$ are model constants. Three of them are related to another constant $C$ by

$$
\begin{aligned}
C_{R P} & =\frac{C+8}{11} \\
C_{R Q} & =\frac{8 C-2}{11} \\
C_{R S} & =\frac{60 C-4}{55}
\end{aligned}
$$

The constant $C$ depends on authors. Table 1 gives two sets of values for the model constants, taken from Launder et al. ${ }^{7}$ and Wallin and Johansson ${ }^{8}$, hereinafter referred to as the LRR and WJ models, respectively. The constants $C_{R R}$ and $C_{R G}$ are taken from Rodi ${ }^{9}$.

We must now focus on the turbulent kinetic energy $k$, which appears necessary to close (12), (14) and (15). Taking the trace of (5) gives

$$
\frac{D k}{D t}=P_{k}+G_{k}+D_{k}-\varepsilon
$$

where $P_{k}, G_{k}, D_{k}$ and $\varepsilon$ are scalar quantities respectively representing production, buoyancy, diffusion and dissipation rate. The first two are explicitely given by

$$
\begin{aligned}
P_{k} & =\frac{1}{2} \operatorname{tr} \mathbf{P}_{\mathbf{R}}=-k \operatorname{tr}(\mathbf{a} \mathbf{S}) \\
G_{k} & =\frac{1}{2} \operatorname{tr} \mathbf{G}_{\mathbf{R}}=-\beta_{T} \mathbf{g} \cdot \mathbf{Q}_{T}
\end{aligned}
$$

while the dissipation $\varepsilon$ requires a model. This is generally achieved through a governing equation similar to (17):

$$
\frac{D \varepsilon}{D t}=P_{\varepsilon}+G_{\varepsilon}+D_{\varepsilon}-\varepsilon_{\varepsilon}
$$

Contrary to (17) however, this equation is purely heuristic (although attempts have been made to derive it on a mathematical background with RNG models $\left.{ }^{10}\right)$. The production, buoyancy and dissipation of $\varepsilon$ are given by

$$
\begin{aligned}
P_{\varepsilon} & =C_{\varepsilon 1} \frac{\varepsilon}{k} P_{k} \\
G_{\varepsilon} & =C_{\varepsilon 3} \frac{\varepsilon}{k} G_{k} \\
\varepsilon_{\varepsilon} & =C_{\varepsilon 2} \frac{\varepsilon^{2}}{k}
\end{aligned}
$$


with model constants $C_{\varepsilon 1}, C_{\varepsilon 2}$ and $C_{\varepsilon 3}$ given by Table 2, from Launder and Spalding ${ }^{11}$ (as mentioned before, the diffusion term $D_{\varepsilon}$ will not be used in the present work).

To finish with the description of equation (5), the dissipation tensor is modelled from an isotropy assumption regarding the smallest (dissipative) turbulent structures. Relating the trace of (5) to (19) thus gives

$$
\varepsilon_{\mathbf{R}}=\frac{2}{3} \varepsilon \mathbf{I}
$$

With (12), (13), (14) and (15) and (21), we may now rewrite (5) in an explicit form. After a few algebra (in particular, using (9) and (18)), we get

$$
\begin{aligned}
\frac{D \mathbf{R}}{D t}-\mathbf{D}_{\mathbf{R}}= & -C_{R R} \varepsilon \mathbf{a}-B_{a 1} k(\mathbf{a S}+\mathbf{S a})+B_{a 2} k(\mathbf{a} \boldsymbol{\Omega}-\mathbf{\Omega a}) \\
& -B_{a 3} k \mathbf{S}+\left[\frac{2}{3}\left(P_{k}+G_{k}-\varepsilon\right)+B_{a 4} k \operatorname{tr}(\mathbf{a S})\right] \mathbf{I} \\
& -B_{a 5} \beta_{T}\left[\left(\mathbf{g} \otimes \mathbf{Q}_{T}\right)^{S}-\frac{1}{3}\left(\mathbf{g} \cdot \mathbf{Q}_{T}\right) \mathbf{I}\right]
\end{aligned}
$$

where

$$
\begin{aligned}
B_{a 1} & =1-C_{R P}-C_{R Q}=\frac{5-9 C}{11} \\
B_{a 2} & =1-C_{R P}+C_{R Q}=\frac{7 C+1}{11} \\
B_{a 3} & =C_{R S}+\frac{4}{3}\left(1-C_{R P}-C_{R Q}\right)=\frac{8}{15} \\
B_{a 4} & =\frac{2}{3}\left(1-C_{R P}-C_{R Q}\right)=\frac{2}{3} B_{a 1}=\frac{10-18 C}{33} \\
B_{a 5} & =2\left(1-C_{R G}\right)
\end{aligned}
$$

The value of these coefficients for the LRR and WJ models are given in Table 3. In section 4, we will construct an approximate solution to (22). However, a model is now required to model the scalar flux $\mathbf{Q}_{T}$, a work which will be done in the coming section.

\subsection{Scalar fluxes}

The governing equation for $\mathbf{Q}_{T}$ can be written as

$$
\frac{D \mathbf{Q}_{T}}{D t}=\mathbf{P}_{T}+\mathbf{G}_{T}+\mathbf{D}_{T}+\boldsymbol{\Phi}_{T}-\varepsilon_{T}
$$

where $\mathbf{P}_{T}, \mathbf{G}_{T}, \mathbf{D}_{T}, \mathbf{\Phi}_{T}$ and $\varepsilon_{T}$ are respectively production, buoyancy, diffusion, pressure-scalar gradient correlation and dissipation vectors. As we did for Reynolds stresses in the previous section, we will now describe the models 
used here. The production is written exactly (from the Navier-Stokes and scalar equations) as

$$
\mathbf{P}_{T}=-\mathbf{R} \boldsymbol{\Gamma}_{T}-\boldsymbol{\Gamma}_{u} \mathbf{Q}_{T}
$$

with the mean scalar gradient

$$
\boldsymbol{\Gamma}_{T}=\nabla \bar{T}=\frac{\partial \bar{T}}{\partial x_{i}} \mathbf{e}_{i}
$$

The buoyancy is explicitely written as

$$
\mathbf{G}_{T}=-\beta_{T} \theta \mathbf{g}
$$

where

$$
\theta=\overline{T^{2}}
$$

is the scalar turbulent fluctuation variance. The pressure-scalar gradient correlation is usually approximated together with the dissipation by the following model: $: 5,9$

$$
\boldsymbol{\Phi}_{T}-\varepsilon_{T}=-C_{T Q} \frac{\varepsilon}{k} \mathbf{Q}_{T}+C_{T \Gamma} \boldsymbol{\Gamma}_{u} \mathbf{Q}_{T}+C_{T \Gamma}^{\prime} \boldsymbol{\Gamma}_{u}^{T} \mathbf{Q}_{T}+C_{T R} \mathbf{R} \boldsymbol{\Gamma}_{T}+C_{T G} \beta_{T} \theta \mathbf{g}
$$

where $C_{T Q}, C_{T \Gamma}, C_{T \Gamma}^{\prime}, C_{T R}$ and $C_{T G}$ are model constants given by Table 4 . Two sets of constants are given here, from Högström et al. ${ }^{12}$ and Wikström et $a l .,{ }^{5}$ respectively referred to as HWJ and WWJ models. Note that in the WWJ model, one of the "constants" depends on quantities are $\tau$ and $\tau_{\theta}$, defined as the characteristic timescales of turbulence corresponding to momentum and and scalar, respectively. We now give a definition of these two parameters. First, as we did for $k$, we can write an equation governing $\theta$ :

$$
\frac{D \theta}{D t}=P_{\theta}+D_{\theta}-\varepsilon_{\theta}
$$

where $P_{\theta}, D_{\theta}$ and $\varepsilon_{\theta}$ are scalar quantities respectively representing production, diffusion and dissipation. In principle, equation (30) requires a closure equation for $\varepsilon_{\theta}$. However, it will not be used in the final EARSM established herein, since we will build a simplified model for $\theta$ in section 4.1. However, it is useful to give the explicit form of the first term of (30):

$$
P_{\theta}=-2 \mathbf{Q}_{T} \cdot \boldsymbol{\Gamma}_{T}
$$

We will also use in the following the timescales $\tau$ and $\tau_{\theta}$, defined by

$$
\begin{aligned}
\tau & =\frac{k}{\varepsilon} \\
\tau_{\theta} & =\frac{\theta}{\varepsilon_{\theta}}
\end{aligned}
$$

As many authors did before, we will assume that the ratio between the two timescales is a constant:

$$
\frac{\tau_{\theta}}{\tau}=R
$$


The value $R=1.1$ was recommended by $\operatorname{Rodi}^{9}$ and is close to the values obtained by DNS by Schwertfirm and Manhart ${ }^{13}$ for passive scalars (although the question of how long this value keeps valid for buoyant flows could raise some difficulties). From these premises, we now estimate the coefficient $C_{T Q}$ of the WWJ model (see Table 4):

$$
C_{T Q}=1.6\left(1+\frac{1}{R}\right)=3.05
$$

Note that the timescale $\tau$ is also governed by an advection-diffusion equation derived from (17) and (19):

$$
\frac{D \tau}{D t}=\left(1-C_{\varepsilon 1}\right) P_{k}^{*}+\left(1-C_{\varepsilon 3}\right) G_{k}^{*}-1+C_{\varepsilon 2}+D_{\tau}
$$

with an appropriate diffusion term $D_{\tau}$, and

$$
\begin{aligned}
P_{k}^{*} & =\frac{P_{k}}{\varepsilon} \\
G_{k}^{*} & =\frac{G_{k}}{\varepsilon}
\end{aligned}
$$

Let us come now back to the clusore of the scalar flux equation. With (25), (27) and (29), equation (24) now reads

$2\left(\frac{D \mathbf{Q}_{T}}{D t}-\mathbf{D}_{T}\right)=-2 C_{T Q} \frac{\varepsilon}{k} \mathbf{Q}_{T}-B_{T 1} \mathbf{S Q}_{T}-B_{T 2} \mathbf{\Omega Q}_{T}-B_{T 3} \mathbf{R} \boldsymbol{\Gamma}_{T}-B_{T 4} \beta_{T} \theta \mathbf{g}$

where

$$
\begin{aligned}
& B_{T 1}=2\left(1-C_{T \Gamma}-C_{T \Gamma}^{\prime}\right) \\
& B_{T 2}=2\left(1-C_{T \Gamma}+C_{T \Gamma}^{\prime}\right) \\
& B_{T 3}=2\left(1-C_{T R}\right) \\
& B_{T 4}=2\left(1-C_{T G}\right)
\end{aligned}
$$

The value of these coefficients for the HWJ and WWJ models are given in Table 5 .

In the coming sections, we will make assumptions in order to transform the set of equations (22) and (37) into a linear system, which will allow us to derive explicit analytical solutions.

\section{Explicit algebraic models}

\subsection{Weak equilibrium and subsequent linear system}

At the heart of EARSM is the assumption of weak equilibrium, which stands that under some conditions ${ }^{6}$ the advection and diffusion terms of turbulent 
fluxes like $\mathbf{R}$ can be related to those of simpler quantities such as $k$. We will first assume two laws of weak equilibrium:

$$
\begin{aligned}
\left(\frac{D \mathbf{R}}{D t}-\mathbf{D}_{\mathbf{R}}\right) k & =\left(\frac{D k}{D t}-D_{k}\right) \mathbf{R} \\
\left(\frac{D \mathbf{Q}_{T}}{D t}-\mathbf{D}_{T}\right) k & =\frac{1}{2}\left(\frac{D k}{D t}-D_{k}\right) \mathbf{Q}_{T}
\end{aligned}
$$

These equations were first stated by $\mathrm{Pope}^{4}$ and Rodi ${ }^{9}$ respectively and remain very classical in the theory of EARSM (with a few variants ${ }^{14,15}$ which would not affect the considerations presented below). As these authors explain, they come from the idea that dimensionless fluxes are in equilibrium. Equation (39), for example, is equivalent to assume

$$
\frac{D \mathbf{a}}{D t}-\mathbf{D}_{\mathbf{a}}=\mathbf{0}
$$

where $\mathbf{D}_{\mathbf{a}}$ is the diffusion of $\mathbf{a}$ obtained from (5). Similarly, one assumes here that the ratio of turbulent kinetic energy to scalar variance satisfies the same equilibrium:

$$
\frac{D(\theta / k)}{D t}-D_{\theta / k}=0
$$

This immediately leads to the following additional weak equilibrium equation:

$$
\left(\frac{D \theta}{D t}-D_{\theta}\right) k=\left(\frac{D k}{D t}-D_{k}\right) \theta
$$

Let us consider now (39) and (40). Together with (17), they give

$$
\begin{aligned}
\frac{D \mathbf{R}}{D t}-\mathbf{D}_{\mathbf{R}} & =\frac{P_{k}+G_{k}-\varepsilon}{k} \mathbf{R} \\
2\left(\frac{D \mathbf{Q}_{T}}{D t}-\mathbf{D}_{T}\right) & =\frac{P_{k}+G_{k}-\varepsilon}{k} \mathbf{Q}_{T}
\end{aligned}
$$

Before constructing our EARSM, we will now write our variables in a dimensionless form. We first introduce dimensionless gradients and fluxes:

$$
\begin{aligned}
\mathbf{S}^{*} & =\tau \mathbf{S} \\
\boldsymbol{\Omega}^{*} & =\tau \boldsymbol{\Omega} \\
\boldsymbol{\Gamma}_{T}^{*} & =\beta_{T} g \tau^{2} \boldsymbol{\Gamma}_{T} \\
\mathbf{Q}_{T}^{*} & =\frac{\beta_{T} g}{\varepsilon} \mathbf{Q}_{T} \\
\theta^{*} & =\left(\beta_{T} g \tau\right)^{2} \frac{\theta}{k}
\end{aligned}
$$

(the stars will always refer to non-dimensional quantities in the following). Then (36) becomes

$$
\begin{aligned}
P_{k}^{*} & =-\operatorname{tr}\left(\mathbf{a S}^{*}\right) \\
G_{k}^{*} & =\mathbf{e}_{z} \cdot \mathbf{Q}_{T}^{*}
\end{aligned}
$$


where $\mathbf{e}_{z}$ is the vertical upwards unit vector. Next, with relations (44) and using (11), (22) and (37) can be rewritten as linear algebraic equations, i.e. containing no time or spatial derivative of the unknowns $\mathbf{a}$ and $\mathbf{Q}_{T}$ :

$$
\begin{aligned}
\mathbf{a} & =C_{a 1}\left(\mathbf{a} \mathbf{S}^{*}+\mathbf{S}^{*} \mathbf{a}\right)+C_{a 2}\left(\mathbf{a} \mathbf{\Omega}^{*}-\mathbf{\Omega}^{*} \mathbf{a}\right)+C_{a 3} \mathbf{S}^{*} \\
& +C_{a 4} \operatorname{tr}\left(\mathbf{a} \mathbf{S}^{*}\right) \mathbf{I}+C_{a 5}\left[\left(\mathbf{e}_{z} \otimes \mathbf{Q}_{T}^{*}\right)^{S}-\frac{1}{3}\left(\mathbf{e}_{z} \cdot \mathbf{Q}_{T}^{*}\right) \mathbf{I}\right] \\
\mathbf{Q}_{T}^{*} & =C_{T 1} \mathbf{S}^{*} \mathbf{Q}_{T}^{*}+C_{T 2} \mathbf{\Omega}^{*} \mathbf{Q}_{T}^{*}+C_{T 3} \mathbf{a} \Gamma_{T}^{*}+\frac{2}{3} C_{T 3} \boldsymbol{\Gamma}_{T}^{*}-C_{T 4} \theta^{*} \mathbf{e}_{z}
\end{aligned}
$$

We have used here a slightly different set of coefficients, defined by

$$
\begin{aligned}
C_{a 1} & =-B_{a 1} C_{a 0} \\
C_{a 2} & =B_{a 2} C_{a 0} \\
C_{a 3} & =-B_{a 3} C_{a 0} \\
C_{a 4} & =B_{a 4} C_{a 0} \\
C_{a 5} & =B_{a 5} C_{a 0}
\end{aligned}
$$

and

$$
\begin{aligned}
& C_{T 1}=-B_{T 1} C_{T 0} \\
& C_{T 2}=-B_{T 2} C_{T 0} \\
& C_{T 3}=-B_{T 3} C_{T 0} \\
& C_{T 4}=-B_{T 4} C_{T 0}
\end{aligned}
$$

where

$$
\begin{aligned}
C_{a 0} & =\frac{1}{P_{k}^{*}+G_{k}^{*}+C_{R R}-1} \\
C_{T 0} & =\frac{1}{P_{k}^{*}+G_{k}^{*}+2 C_{T Q}-1}
\end{aligned}
$$

By giving algebraic equations, the approximation made presents the advantage to avoid the use of boundary conditions for $\mathbf{a}$ and $\mathbf{Q}_{T}^{*}$ in a numerical model. In order to search solutions $\left(\mathbf{a}, \mathbf{Q}_{T}^{*}\right)$ of $(47)$, we will now restrict our considerations to 2-D vertical flows, i.e. flows which are (statistically) invariant along an axis called $y$ (spanwise), with zero mean velocities along $y$. Under these assumtions, the spatial derivative $\partial / \partial y$ play no role. Therefore, the dimensionless 
gradients may be written as

$$
\begin{aligned}
\mathbf{S}^{*} & =\left(\begin{array}{ccc}
S_{x x}^{*} & 0 & S_{x z}^{*} \\
0 & 0 & 0 \\
S_{x z}^{*} & 0 & -S_{x x}^{*}
\end{array}\right) \\
\boldsymbol{\Omega}^{*} & =\Omega_{x z}^{*}\left(\begin{array}{ccc}
0 & 0 & 1 \\
0 & 0 & 0 \\
-1 & 0 & 0
\end{array}\right) \\
\boldsymbol{\Gamma}_{T}^{*} & =\left(\begin{array}{c}
\Gamma_{T, x}^{*} \\
0 \\
\Gamma_{T, z}^{*}
\end{array}\right)
\end{aligned}
$$

In the Reynolds-averaged Navier-Stokes momentum equation (first equation of (1)), the term $\nabla \cdot \mathbf{R}$ then shows that only the components $a_{x x}, a_{x z}$ and $a_{z z}$ are required to close the momentum equation, while some terms are identically null by symmetry ${ }^{6}$. Indeed, $\mathbf{a}$ is symmetric and traceless, thus it can be written

$$
\mathbf{a}=\left(\begin{array}{ccc}
a_{x x} & 0 & a_{x z} \\
0 & -a_{x x}-a_{z z} & 0 \\
a_{x z} & 0 & a_{z z}
\end{array}\right)
$$

It should be noted that although the central term $-a_{x x}-a_{z z}$ play no role on the 2-D equations, it is non-zero. Indeed, although we made assumptions of two-dimensionality, turbulent fluctuations exist on the $y$-axis. We write it explicitely in (52) to insist on the fact that $\mathbf{a}$ is traceless. Therefore, we see that a has three degrees of freedom and thus must be searched in a threedimensional space. Hence, following Pope ${ }^{4}$ we consider the following basis of the 2-D symmetric traceless tensors:

$$
\begin{aligned}
& \mathbf{T}^{(1)}=\mathbf{S}^{*} \\
& \mathbf{T}^{(2)}=\mathbf{S}^{*} \boldsymbol{\Omega}^{*}-\boldsymbol{\Omega}^{*} \mathbf{S}^{*}=2 \mathbf{S}^{*} \boldsymbol{\Omega}^{*} \\
& \mathbf{T}^{(3)}=\left(\mathbf{S}^{* 2}\right)^{D}
\end{aligned}
$$

(the property $\mathbf{S}^{*} \boldsymbol{\Omega}^{*}=-\boldsymbol{\Omega}^{*} \mathbf{S}^{*}$ only stands in $2-\mathrm{D}$ ). We will then search a as a linear combination of the tensors $\left\{\mathbf{T}^{(i)}\right\}$ :

$$
\mathbf{a}=\alpha \mathbf{S}^{*}+2 \beta \mathbf{S}^{*} \boldsymbol{\Omega}^{*}+\gamma\left(\mathbf{S}^{* 2}\right)^{D}
$$

where $\alpha, \beta$ and $\gamma$ are unknown coefficients. In the right-hand-side of (54), the first term corresponds to Boussinesq's (linear) eddy viscosity model (see section 4.2), while the other two are non-linear. Note that the first term will appear later as slightly non-linear two, since $\alpha, \beta$ and $\gamma$ are functions on the scalar invariants of $\mathbf{S}^{*}$ and $\boldsymbol{\Omega}^{*}$. Similarly, we note that $\mathbf{Q}_{T}^{*}$ has now two required degrees of freedom:

$$
\mathbf{Q}_{T}^{*}=\left(\begin{array}{c}
Q_{T, x}^{*} \\
0 \\
Q_{T, z}^{*}
\end{array}\right)
$$


and must then be searched in a two-dimensional space of vectors. A straightforward basis of this space is

$$
\begin{aligned}
\mathbf{V}^{(1)} & =\boldsymbol{\Gamma}_{T}^{*} \\
\mathbf{V}^{(2)} & =\boldsymbol{\Omega}^{*} \boldsymbol{\Gamma}_{T}^{*}
\end{aligned}
$$

Then we will write

$$
\mathbf{Q}_{T}^{*}=\xi \boldsymbol{\Gamma}_{T}^{*}+\zeta \boldsymbol{\Omega}^{*} \boldsymbol{\Gamma}_{T}^{*}
$$

where $\xi$ and $\zeta$ are unknowns. The first term of the right-hand-side of (57) is the traditional law of Fourier for scalar fluxes (see section 4.2). With these notations, we can first propose a model to estimate $\theta^{*}$ (similarly to Galperin et $\left.a l .{ }^{16}\right)$. From (17), (30) and (43), we can write

$$
\frac{P_{k}+G_{k}-\varepsilon_{k}}{k} \theta=P_{\theta}-\varepsilon_{\theta}
$$

Then, with (33) and (45) we get

$$
\theta^{*}=R\left(\beta_{T} g \tau\right)^{2} \frac{\varepsilon_{\theta}}{\varepsilon}
$$

Combining the last two equations gives

$$
\theta^{*}=-2 R^{\prime} \xi \Gamma_{T}^{* 2}
$$

with

$$
R^{\prime}=\frac{R}{1+R\left(P_{k}^{*}+G_{k}^{*}-1\right)}
$$

We observe that, according to (60) and noting that $\theta^{*}$ is positive by definition, $\xi$ must be negative. This is consistent with (57), since $\mathbf{Q}_{T}^{*} \cdot \boldsymbol{\Gamma}_{T}^{*}$ must be negative according to Fourier's law.

The next step is to find the set of five parameters $(\alpha, \beta, \gamma, \xi, \zeta)$. This is achieved by introducing (54) and (57) into the system (47); this is the key idea of EARSM. This has been done by many authors in $2-\mathrm{D}^{4}$ and $3-\mathrm{D}^{17}$ for nonbuoyant flows. However, in most of the publications concerning active scalars, this work is not performed in the right way. Hattori et al. ${ }^{15}$, for example, added a fourth tensor to the basis (53), namely $\mathbf{G}^{D}$. This seems to be justified by the presence of the buoyant tensor $\mathbf{e}_{z} \otimes \mathbf{Q}_{T}^{*}$ in the momentum equation and the terms involving $\mathbf{Q}_{T}^{*}$ in the scalar equation, but as we already mentioned, this approach is wrong since $\mathbf{a}$ is to be found in a three-dimensional space. The correct method thus consists of developing the buoyant terms of (47) on the respective basis (53) and (56). To achieve this purpose, we first note that the basis (56) is orthogonal. Hence, the decomposition of an arbitrary vector $\mathbf{A}$ on a basis of vectors $\left\{\mathbf{V}^{(i)}\right\}$ is simply given by

$$
\mathbf{A}=\sum_{i} \frac{\mathbf{A} \cdot \mathbf{V}^{(i)}}{\left\|\mathbf{V}^{(i)}\right\|^{2}} \mathbf{V}^{(i)}
$$


Similarly, the basis (53) is orthogonal in the sense that for $i \neq j$ we have $\mathbf{T}^{(i)}: \mathbf{T}^{(j)}=T_{k l}^{(i)} T_{l k}^{(j)}=0$. Thus, the decomposition of a tensor $\mathbf{A}$ on the basis $\left\{\mathbf{T}^{(i)}\right\}$ is

$$
\mathbf{A}=\sum_{i} \frac{\mathbf{A}: \mathbf{T}^{(i)}}{\left\|\mathbf{T}^{(i)}\right\|^{2}} \mathbf{T}^{(i)}
$$

In the above two formulae, we used scalar products and norms of vectors $\mathbf{A}$ and $\mathbf{B}$, naturally defined by

$$
\begin{aligned}
\mathbf{A} \cdot \mathbf{B} & =A_{i} B_{i} \\
\|\mathbf{A}\|^{2} & =\mathbf{A} \cdot \mathbf{A}=A_{i} A_{i}
\end{aligned}
$$

and for tensors:

$$
\begin{aligned}
\mathbf{A} & : \quad \mathbf{B}=\operatorname{tr}(\mathbf{A B})=A_{i j} B_{j i} \\
\|\mathbf{A}\|^{2} & =\mathbf{A}: \mathbf{A}=A_{i j} A_{j i}
\end{aligned}
$$

As an example, we may define the (dimensionless) mean scalar rate-of-strain $S^{*}$ and mean scalar vorticity $\Omega^{*}$, as well as the (dimensionless) mean active scalar gradient $\Gamma_{T}^{*}$ by

$$
\begin{aligned}
S^{*} & =\sqrt{2}\left\|\mathbf{S}^{*}\right\| \\
\Omega^{*} & =\sqrt{2}\left\|\boldsymbol{\Omega}^{*}\right\|=2\left|\Omega_{x z}^{*}\right| \\
\Gamma_{T}^{*} & =\left\|\boldsymbol{\Gamma}_{T}^{*}\right\|
\end{aligned}
$$

Introducing (57) into (47) then leads to four new vectors, whose decompositions are given through (62), after a few algebra, by

$$
\begin{aligned}
\mathbf{S}^{*} \boldsymbol{\Gamma}_{T}^{*} & =S_{1} \mathbf{V}^{(1)}+\frac{4 S_{2}}{\Omega^{* 2}} \mathbf{V}^{(2)} \\
\mathbf{S}^{*} \boldsymbol{\Omega}^{*} \boldsymbol{\Gamma}_{T}^{*} & =S_{2} \mathbf{V}^{(1)}-S_{1} \mathbf{V}^{(2)} \\
\boldsymbol{\Omega}^{* 2} \boldsymbol{\Gamma}_{T}^{*} & =-\frac{\Omega^{* 2}}{4} \mathbf{V}^{(1)} \\
\Gamma_{T}^{* 2} \mathbf{e}_{z} & =\Gamma_{1} \mathbf{V}^{(1)}+\frac{4 \Gamma_{2}}{\Omega^{* 2}} \mathbf{V}^{(2)}
\end{aligned}
$$

(see Appendix 1 for the detailed derivation of the second term, as an example). In equation (67) appear four new scalar quantities defined by

$$
\begin{aligned}
& S_{1}=\frac{\boldsymbol{\Gamma}_{T}^{* T} \mathbf{S}^{*} \boldsymbol{\Gamma}_{T}^{*}}{\Gamma_{T}^{* 2}}=\frac{\Gamma_{T, i}^{*} S_{i j}^{*} \Gamma_{T, j}^{*}}{\Gamma_{T, m m}^{*}} \\
& S_{2}=\frac{\boldsymbol{\Gamma}_{T}^{* T} \mathbf{S}^{*} \boldsymbol{\Omega}^{*} \boldsymbol{\Gamma}_{T}^{*}}{\Gamma_{T}^{* 2}}=\frac{\Gamma_{T, i}^{*} S_{i j}^{*} \Omega_{j k}^{*} \Gamma_{T, k}^{*}}{\Gamma_{T, m m}^{*}} \\
& \Gamma_{1}=\mathbf{e}_{z} \cdot \boldsymbol{\Gamma}_{T}^{*}=\Gamma_{T, z}^{*} \\
& \Gamma_{2}=\mathbf{e}_{z}^{T} \boldsymbol{\Omega}^{*} \boldsymbol{\Gamma}_{T}^{*}=\frac{1}{2} \Omega^{*} \Gamma_{T, x}^{*}
\end{aligned}
$$


Next, introducing (54) into (47) lead to other (tensorial) unkonwns, whose decomposition is similarly performed using (63). We will not detail here the numerous steps of algebra needed to achieve these decomposition (see Appendix 2 , where an example is given). The results are all based on the scalar quantities (66) and (68), and lead to a system of five linear equations in $(\alpha, \beta, \gamma, \xi, \zeta)$, which may be summarized as

$$
\mathbf{M A}=\mathbf{B}
$$

with the following two five-dimensional vectors:

$$
\begin{aligned}
& \mathbf{A}=(\alpha, \beta, \gamma, \xi, \zeta)^{T} \\
& \mathbf{B}=\left(-C_{a 3}, 0,0,-\frac{2 C_{T 3}}{3}, 0\right)^{T}
\end{aligned}
$$

and the $5 \times 5$ following matrix:

$$
\mathbf{M}=\left(\begin{array}{ccccc}
-1 & -C_{a 2} \Omega^{* 2} & \frac{C_{a 1} S^{* 2}}{6} & \frac{2 C_{a 5}}{S^{* 2}}\left(S_{1} \Gamma_{1}+\frac{4 S_{2} \Gamma_{2}}{\Omega^{* 2}}\right) & \frac{2 C_{a 5}}{S^{* 2}}\left(S_{2} \Gamma_{1}-S_{1} \Gamma_{2}\right) \\
C_{a 2} & -1 & 0 & \frac{4 C_{a 5}}{S^{* 2} \Omega^{* 2}}\left(S_{2} \Gamma_{1}-S_{1} \Gamma_{2}\right) & -\frac{C_{a 5}}{S^{* 2}}\left(S_{1} \Gamma_{1}+\frac{4 S_{2} \Gamma_{2}}{\Omega^{* 2}}\right) \\
2 C_{a 1} & 0 & -1 & \frac{2 C_{a 5} \Gamma_{1}}{S^{* 2}} & C_{T 1} \\
C_{T 3} S_{1} & 2 C_{T 3} S_{2} & \frac{C_{T 3} S^{* 2}}{12} & -1+C_{T 1} S_{1}+2 R^{\prime} C_{T 4} \Gamma_{1} & C_{T 1} S_{2}-\frac{C_{T 2} \Omega^{* 2}}{4} \\
\frac{4 C_{T 3} S_{2}}{\Omega^{* 2}} & -2 C_{T 3} S_{1} & 0 & C_{T 2}+\frac{4 C_{T 1} S_{2}}{\Omega^{* 2}}+\frac{8 R^{\prime} C_{T 4} \Gamma_{2}}{\Omega^{* 2}} & -\left(1+C_{T 1} S_{1}\right)
\end{array}\right)
$$

We may note that the scalar gradient appears in (71) through the four dimensionless numbers $S_{1}, S_{2}, \Gamma_{1}$ and $\Gamma_{2}$ defined by (76). Thus, the traditional approach consisting of modelling the stratification effects with one single dimensionless number, namely the Richardson number (see next section), is not correct for the general case of 2-D flows.

The system (69)-(71) could be exactly solved, but the algebraic solution would certainly be too complicated to be used with benefit in a numerical code. However, particular cases can be treated more easily. Let us first consider the case of a passive scalar (i.e. $\beta_{T}=0$, or $C_{a 5}=C_{T 4}=0$ ). With model WJ for Reynolds stresses (i.e. $C_{a 1}=0$, according to Table 3 and definition (48)) and model WWJ for scalar fluxes (see Table 2 and (49)), the matrix turns to

$$
\mathbf{M}=\left(\begin{array}{ccccc}
-1 & -C_{a 2} \Omega^{* 2} & 0 & 0 & 0 \\
C_{a 2} & -1 & 0 & 0 & 0 \\
0 & 0 & -1 & 0 & 0 \\
-2 C_{T 0} S_{1} & -4 C_{T 0} S_{2} & -\frac{C_{T 0}}{6} S^{* 2} & -1-2 C_{T 0} S_{1} & -2 C_{T 0} S_{2}+\frac{C_{T 0}}{2} \Omega^{* 2} \\
-\frac{8 C_{T 0}}{\Omega^{* 2}} S_{2} & 4 C_{T 0} S_{1} & 0 & -2 C_{T 0}-\frac{8 C_{T 0}}{\Omega^{* 2}} S_{2} & -1+2 C_{T 0} S_{1}
\end{array}\right)
$$

and

$$
\mathbf{B}=\left(-C_{a 3}, 0,0, \frac{4 C_{T 0}}{3}, 0\right)^{T}
$$

The scalar is thus completely separated from the flow, as required. The buoyancy $G_{k}^{*}$ is null; according to (50) the coefficients $C_{a 0}$ and $C_{T 0}$ are thus 
now defined by

$$
\begin{aligned}
C_{a 0} & =\frac{1}{P_{k}^{*}+C_{R R}-1} \\
C_{T 0} & =\frac{1}{P_{k}^{*}+2 C_{T Q}-1}
\end{aligned}
$$

The solution to (69) now reads

$$
\begin{aligned}
\alpha & =\frac{C_{a 3}}{H_{1}} \\
\beta & =\frac{C_{a 2} C_{a 3}}{H_{1}} \\
\gamma & =0 \\
\xi & =-\frac{2 C_{T 0}}{3 H_{1} H_{2}}\left(H_{3} S_{1}+2 H_{4} S_{2}+2 H_{1}-H_{5}\right) \\
\zeta & =\frac{2 C_{T 0}}{3 H_{1} H_{2}}\left(2 H_{4} S_{1}-\frac{4}{\Omega^{* 2}} H_{3} S_{2}+2 C_{a 2} H_{5}+4 C_{T 0} H_{1}\right)
\end{aligned}
$$

with

$$
\begin{aligned}
& H_{1}=1+C_{a 2}^{2} \Omega^{* 2} \\
& H_{2}=1+C_{T 0}^{2}\left(\Omega^{* 2}-S^{* 2}\right) \\
& H_{3}=3 C_{a 3}-4 C_{T 0}-C_{a 2} C_{T 0}\left(4 C_{a 2}+3 C_{a 3}\right) \Omega^{* 2} \\
& H_{4}=3 C_{a 3}\left(C_{a 2}+C_{T 0}\right) \\
& H_{5}=\frac{3}{2} C_{a 3} C_{T 0} S^{* 2}
\end{aligned}
$$

The first three equations of (75) correspond to the EARSM proposed by Wallin and Johansson ${ }^{8}$ for clear flow (i.e. without density variation due to active scalar). Their model is closed by estimating the production $P_{k}^{*}$ from the first equation of (46), giving with (54), (66), (75) and (76):

$$
P_{k}^{*}=-\frac{\alpha}{2} S^{* 2}=-\frac{C_{a 3}}{2} \frac{S^{* 2}}{1+C_{a 2}^{2} \Omega^{* 2}}
$$

Since $C_{a 2}$ and $C_{a 3}$ are related to $P_{k}^{*}$ through (48) and (74), the formula (77) yields a more complex (implicit) relation for $P_{k}^{*}$, taking the form of a third order polynomial equation, the solution of which is given by Girimaji ${ }^{18}$. We see that our model turns back to a well-known model in the particular case of clear flows. The last two equations of (75), together with (57), also give an algebraic expression of the turbulent fluxes for the passive scalar. Other attempts have been made to derive explicit algebraic turbulent flux models for passive scalars in the past. In particular, Wikström et al. ${ }^{5}$ give a more complex model with validations in several standard cases; it can be shown that our model given by (75) and (76) is mathematically equivalent. However, it is not on the purpose 
of this work to investigate in details the model obtained here for passive scalar (this will be the purpose of a future paper); on the contrary we will now focus on the active scalar case for shear flows (see next section). However, it should be emphasized that the approach proposed here looks more rational than the method proposed by Wallin and Johansson, since the linear system (47) is solved for Reynolds stresses and turbulent scalar fluxes together. The present approach could be called an Explicit Algebraic Reynolds Stress and Scalar Flux Model (EARSSFM).

\subsection{Solution for simply stratified flows}

From now on (except at the beginning of section 5.3) we focus on the case of simple shear flows, i.e. flows following one direction (herein denoted by $x$ ) and varying along the other direction $z$ (we still consider 2-D flows only). In particular, we limit our considerations to stable stratifications, so that natural convection do not exist (no vertical mean velocities). Many flows showing such properties are encountered in industrial and environmental fluid dynamics: heated pipe flow, atmospheric boundary layer, estuaries, etc. With this additional assumption, the quantities appearing in the matrix (71) simplify to:

$$
\begin{aligned}
\Omega^{*} & =S^{*}=\tau\left|\frac{\partial \bar{u}}{\partial z}\right| \\
\Gamma_{T}^{*} & =\beta_{T} g \tau^{2}\left|\frac{\partial \bar{T}}{\partial z}\right| \\
S_{1} & =0 \\
S_{2} & =\frac{S^{* 2}}{4} \\
\Gamma_{1} & =\Gamma_{T}^{*} \\
\Gamma_{2} & =0
\end{aligned}
$$

where $\bar{u}$ is the mean velocity component along $x$. Moreover, the gradients now appear in the matrix through a single dimensionless parameter, namely $\Gamma_{1} / S_{2}$. We may thus introduce the gradient Richardson number, measuring the degree of density stratification:

$$
R i=\frac{\Gamma_{T}^{*}}{S^{* 2}}=\frac{\beta_{T} g\left|\frac{\partial \bar{T}}{\partial z}\right|}{\left(\frac{\partial \bar{u}}{\partial z}\right)^{2}}
$$

(with our assomption of stable stratification, $R i$ is non-negative). This notation allows us to write $\mathbf{M}$ in the simplified form

$$
\mathbf{M}=\left(\begin{array}{ccccc}
-1 & -C_{a 2} S^{* 2} & \frac{C_{a 1}}{6} S^{* 2} & 0 & \frac{C_{a 5}}{2} S^{* 2} R i \\
C_{a 2} & -1 & 0 & C_{a 5} R i & 0 \\
2 C_{a 1} & 0 & -1 & 2 C_{a 5} R i & 0 \\
0 & \frac{C_{T 3}}{2} S^{* 2} & \frac{C_{T 3}}{12} S^{* 2} & -1+2 R^{\prime} C_{T 4} S^{* 2} R i & \frac{C_{T 1}-C_{T 2}}{4} S^{* 2} \\
C_{T 3} & 0 & 0 & C_{T 1}+C_{T 2} & -1
\end{array}\right)
$$


With model WJ for stresses and model WWJ for scalar fluxes (both prefered from now on), it turns into

$$
\mathbf{M}=\left(\begin{array}{ccccc}
-1 & -C_{a 2} S^{* 2} & 0 & 0 & \frac{C_{a 5}}{2} S^{* 2} R i \\
C_{a 2} & -1 & 0 & C_{a 5} R i & 0 \\
0 & 0 & -1 & 2 C_{a 5} R i & 0 \\
0 & -C_{T 0} S^{* 2} & -\frac{C_{T 0}}{6} S^{* 2} & -1-2 R^{\prime} C_{T 0} S^{* 2} R i & 0 \\
-2 C_{T 0} & 0 & 0 & -4 C_{T 0} & -1
\end{array}\right)
$$

The solution to the linear system (69) is now given by

$$
\begin{aligned}
\alpha & =\frac{K_{1}}{D} \\
\beta & =\frac{1}{D}\left(C_{a 2} K_{1}-C_{a 5} K_{2} R i\right) \\
\gamma & =-\frac{2 C_{a 5} K_{2}}{D} R i \\
\xi & =-\frac{K_{2}}{D} \\
\zeta & =\frac{2 C_{T 0}}{D}\left(2 K_{2}-K_{1}\right)
\end{aligned}
$$

with

$$
D=\frac{1}{4}\left[\left(\frac{3}{C_{T 0}}+2 R^{\prime \prime} \Gamma_{T}^{*}\right) K_{2}-3 C_{a 2} K_{1} S^{* 2}\right]
$$

and

$$
\begin{aligned}
& K_{1}=3 C_{a 3}+2 C_{T 0}\left[2 C_{a 5}\left(C_{a 2}+2 C_{T 0}\right)+C_{a 3} R^{\prime \prime}\right] \Gamma_{T}^{*} \\
& K_{2}=C_{T 0}\left[\left(4 C_{a 2}+3 C_{a 3}\right) C_{a 2} S^{* 2}+4\left(C_{a 5} C_{T 0} \Gamma_{T}^{*}+1\right)\right] \\
& R^{\prime \prime}=2 C_{a 5}-3 R^{\prime}
\end{aligned}
$$

It can be easily verified that equations (82) to (84) give, for a passive scalar $\left(C_{a 5}=R^{\prime}=0\right)$, the following particular solution:

$$
\begin{aligned}
\alpha & =\frac{C_{a 3}}{H_{1}^{\prime}} \\
\beta & =\frac{C_{a 2} C_{a 3}}{H_{1}^{\prime}} \\
\gamma & =0 \\
\xi & =-\frac{H_{3}^{\prime}}{3 H_{1}^{\prime}} \\
\zeta & =\frac{2 C_{T 0}}{3 H_{1}^{\prime}}\left(2 H_{3}^{\prime}-3 C_{a 3}\right)
\end{aligned}
$$

with

$$
\begin{aligned}
& H_{1}^{\prime}=1+C_{a 2}^{2} S^{* 2} \\
& H_{3}^{\prime}=C_{T 0}\left[C_{a 2}\left(4 C_{a 2}+3 C_{a 3}\right) S^{* 2}+4\right]
\end{aligned}
$$


which can also be obtained from the general passive scalar solution (75) and (76) with conditions (78). Equations (82) to (84) could be used in a CFD code without any changes. However, one should keep in mind that the coefficients $C_{a 2}, C_{a 3}$, etc. depend on $P_{k}^{*}+G_{k}^{*}$ through (50). We have seen before that $P_{k}^{*}$ is given by (77); similarly the second equation of (46), together with (57) and (66), gives

$$
G_{k}^{*}=\xi \Gamma_{T}^{*}
$$

With (77), (82), (83) and (84), (87) would give an implicit relation in $P_{k}^{*}+G_{k}^{*}$ to close model. Unfortunately, this process leads to a five order polynomial equation, which is known to have no analytical solution. In a numerical code, it is thus certainly simpler to use (77) and (87) in order to estimate $P_{k}^{*}$ and $G_{k}^{*}$ from the values of $\alpha$ and $\xi$ obtained at the previous time step.

For practical applications, the model should be simplified through an assumption giving $P_{k}^{*}+G_{k}^{*}$ explicitely, together with $S^{*}$ and $\Gamma_{T}^{*}$, both implicit functions of $R i$ through the governing equations (1). This can be achieved by assuming ${ }^{16}$ that advection and diffusion of $k$ can be neglected in (17), leading to the well-known equilibrium

$$
P_{k}^{*}+G_{k}^{*}=1
$$

Note that this approximation, together with (17), makes the right-hand-sides of (39) to (??) vanish. Then the coefficients $C_{a 0}, C_{T 0}$ and $R^{\prime}$ defined by (50) and (61) reduce to constants:

$$
\begin{aligned}
C_{a 0} & =\frac{1}{C_{R R}} \\
C_{T 0} & =\frac{1}{2 C_{T Q}} \\
R^{\prime} & =R
\end{aligned}
$$

and thus

$$
R^{\prime \prime}=2 C_{a 5}-3 R
$$

Using (23), (33), (48) and Table 1, the numerical constants of the model (82)-(84) are now given in Table 6.

Using (77), (79) and (87), (88) gives the mean gradients as

$$
\begin{aligned}
S^{* 2} & =\frac{2}{2 \xi R i-\alpha} \\
\Gamma_{T}^{*} & =\frac{2 R i}{2 \xi R i-\alpha}
\end{aligned}
$$

The formulae (91) can be introduced into (82). This gives two non-linear equations with unknowns $\alpha$ and $\xi$, giving the following solution:

$$
\begin{aligned}
\alpha & =\frac{A}{D^{\prime}}\left(A_{\alpha 2} A+2 A_{\alpha 1} R i\right) \\
\xi & =\frac{A}{D^{\prime}}\left(A_{\xi 3} A+2 A_{\xi 1}+2 A_{\xi 2} R i\right)
\end{aligned}
$$


where

$$
\begin{aligned}
D^{\prime} & =\left(2 A_{\xi 3} R i-A_{\alpha 2}\right) A+2\left(2 A_{\xi 2} R i-A_{\alpha 1}+2 A_{\xi 1}\right) R i \\
A & =B_{A 1} R i+B_{A 2}+\sqrt{B_{A 3} R i^{2}+B_{A 4} R i+B_{A 2}^{2}}
\end{aligned}
$$

The constants appearing in (92) and (93) are given by

$$
\begin{aligned}
& A_{\alpha 1}=-C_{T 0}\left[4 C_{a 5}\left(2 C_{T 0}+C_{a 2}+2 C_{a 3}\right)-2 C_{a 3} R^{\prime \prime}\right] \\
& A_{\alpha 2}=-3 C_{a 3} \\
& A_{\xi 1}=C_{a 2} C_{T 0}\left(4 C_{a 2}+3 C_{a 3}\right) \\
& A_{\xi 2}=4 C_{a 5} C_{T 0}^{2} \\
& A_{\xi 3}=4 C_{T 0}
\end{aligned}
$$

and

$$
\begin{aligned}
B_{A 1} & =-\frac{1}{3}\left(A_{D 4}+A_{\xi 3}\right) \\
B_{A 2} & =\frac{1}{6}\left(A_{\alpha 2}-2 A_{D 2}\right) \\
B_{A 3} & =B_{A 1}^{2}-\frac{4}{3}\left(A_{D 3}+A_{\xi 2}\right) \\
B_{A 4} & =2 B_{A 1} B_{A 2}-\frac{2}{3}\left(2 A_{D 1}-A_{\alpha 1}+2 A_{\xi 1}\right)
\end{aligned}
$$

with

$$
\begin{aligned}
& A_{D 1}=C_{a 2} C_{T 0}\left[C_{a 5}\left(5 C_{a 2}-6 C_{T 0}\right)-2 C_{a 2} R^{\prime \prime}\right] \\
& A_{D 2}=3 C_{a 2}^{2} \\
& A_{D 3}=2 C_{a 5} C_{T 0}^{2}\left(4 C_{a 5}-R^{\prime \prime}\right) \\
& A_{D 4}=C_{T 0}\left(11 C_{a 5}-2 R^{\prime \prime}\right)
\end{aligned}
$$

The numerical values of these new coefficients are shown in Table 7.

The quantity $A$ appearing in (93) has the following physical meaning:

$$
A=\frac{2}{S^{* 2}}
$$

and thus must be positive (note that the argument in the squareroot of (93) is always positive). This leads to the following condition:

$$
R i \leq-\frac{2 A_{D 1}-A_{\alpha 1}+2 A_{\xi 1}}{2\left(A_{D 3}+A_{\xi 2}\right)}=0.284=R i_{c}
$$

(reminding that $R i$ is positive in our model). Thus, the present model exhibits a critical value $R i_{c}$ above which the critical Richardson number cannot stand. For $R i=R i_{c}$, it is found that $A=0$, so that $\alpha=\xi=0$ according to (92); 
the turbulent diffusion is thus stopped. This phenomenon, already adressed in many publications, is known as turbulence destruction by buoyancy. In principle, it means that when the density stratification is high enough, the damping of turbulence is so strong that turbulence locally dissapears. Many works have been attempted in order to determine $R i_{c}$ from theoretical considerations, and most of them reached the value $R i_{c}=0.25$ (see e.g. Miles and Howard ${ }^{19}$ ), while Mellor and Yamada ${ }^{2}$ give $R i_{c}=0.21$ and Yamada ${ }^{20} R i_{c}=0.27$. However, the question of the existence of a critical Richardson number is not completely solved, and recent publications suggest that it does not exist. We will investigate that point in section 5.2 .

The present model (92) to (96) is very similar to those of Mellor and Yamada ${ }^{21}$ and Galperin et al. ${ }^{16}$, although it was derived from slightly different considerations. In particular, it is intersting to see how this solution arises from an EARSSFM. The coefficients $\beta, \gamma$, and $\zeta$ can easily be written from (82); however, for simplicity we will not give here their analytical expressions. However, it is easy to give the values of all coefficients for $R i=0$ (passive scalar). Referring to $\alpha_{0}$ as $\alpha(R i=0)$, etc., we find

$$
\begin{aligned}
\alpha_{0} & =2 C_{a 2}^{2}+C_{a 3} \\
\beta_{0} & =C_{a 2}\left(2 C_{a 2}^{2}+C_{a 3}\right) \\
\gamma_{0} & =0 \\
\xi_{0} & =\frac{2 C_{T 0}}{3}\left(3 C_{a 2}-2\right) \\
\zeta_{0} & =\frac{2 C_{T 0}}{3}\left[4 C_{T 0}\left(2-3 C_{a 2}\right)-3\left(2 C_{a 2}^{2}+C_{a 3}\right)\right]
\end{aligned}
$$

in accordance to (85) and (86) with (91).

\section{Performances of the model}

\subsection{Damping functions}

The most famous first order closure models are the Boussinesq eddy-viscosity model (for Reynolds stresses) and the Fourier model (for scalar flux), which read

$$
\begin{gathered}
\mathbf{R}=\frac{2}{3} k \mathbf{I}-2 \nu_{T} \mathbf{S} \\
\mathbf{Q}_{T}=-K_{T} \boldsymbol{\Gamma}_{T}
\end{gathered}
$$

where $\nu_{T}$ and $K_{T}$ are the eddy viscosity and the scalar eddy diffusivity, respectively. From dimensionless considerations, it is known that both quantities 
may be written as

$$
\begin{aligned}
\nu_{T} & =C_{\mu} \frac{k^{2}}{\varepsilon} \\
K_{T} & =C_{\nu} \frac{k^{2}}{\varepsilon}=\frac{\nu_{T}}{\operatorname{Pr}_{T}}
\end{aligned}
$$

where $C_{\mu}$ and $C_{\nu}$ are empirical constants. The quantity $\operatorname{Pr}_{T}$, defined by the second equation of (101), is the turbulent Prandtl number. As pointed out in section 4.1, our models (54) and (57) contain the models (101) and a bit more. From these equations it appears that $C_{\mu}, C_{\nu}$ and $\operatorname{Pr}_{T}$ are no longer constants but vary according to

$$
\begin{aligned}
C_{\mu} & =-\frac{\alpha}{2} \\
C_{\nu} & =-\xi \\
\operatorname{Pr}_{T} & =\frac{C_{\mu}}{C_{\nu}}=\frac{\alpha}{2 \xi}
\end{aligned}
$$

For a passive scalar $(R i=0)$, the quantity $A$ of equation (93) take the following value:

$$
A_{0}=A(R i=0)=-\left(2 C_{a 2}^{2}+C_{a 3}\right)=0.181
$$

and, from (99):

$$
\begin{aligned}
C_{\mu, 0} & =C_{\mu}(R i=0)=-\frac{2 C_{a 2}^{2}+C_{a 3}}{2} \\
C_{\nu, 0} & =C_{\nu}(R i=0)=-\frac{2 C_{T 0}}{3}\left(3 C_{a 2}-2\right) \\
\operatorname{Pr}_{T, 0} & =\operatorname{Pr}_{T}(R i=0)=\frac{C_{\mu, 0}}{C_{\nu, 0}}=\frac{3}{4 C_{T 0}} \frac{2 C_{a 2}^{2}+C_{a 3}}{3 C_{a 2}-2}
\end{aligned}
$$

Numerically, with the values of Table 6 , we find

$$
\begin{gathered}
C_{\mu, 0}=0.090 \\
C_{\nu, 0}=0.121 \\
\operatorname{Pr}_{T, 0}=0.743
\end{gathered}
$$

Wallin and Johansson ${ }^{8}$ had already reported that their EARSM correctly predicts the value of $C_{\mu}$ for simple shear flows without scalar, together with the equality $P_{k}^{*}=1$ (similar to (88)), and it is not surprising to come to the same conclusion since our model turns back to their for a passive scalar, as already mentioned at the end of section 4.1. We also find that the neutral turbulent Prandtl number is very close to the values proposed by various authors on experimental, theoretical or numerical basis: $\operatorname{Pr}_{T, 0}=0.71$ (Turner, ${ }^{22}$ experimental), $\operatorname{Pr}_{T, 0}=0.72$ (Sukoriansky et al., ${ }^{23}$ theoretical), $\operatorname{Pr}_{T, 0}=0.75$ 
(Mellor and Yamada, ${ }^{2}$ theoretical), $\operatorname{Pr}_{T, 0}=0.74$ (Galland, ${ }^{24}$ numerical model with second-order turbulent closure).

We then define the damping functions as

$$
\begin{gathered}
f_{C_{\mu}}(R i)=\frac{C_{\mu}}{C_{\mu, 0}} \\
f_{C_{\nu}}(R i)=\frac{C_{\nu}}{C_{\nu, 0}}
\end{gathered}
$$

The effect of stratification is to reduce (stable) turbulent diffusion, thus these functions must be less than unity and satisfy $f_{C_{\mu}}(0)=f_{C_{\nu}}(0)=1$. It also follows from the observation made in section 4.1 that these function are positive. To avoid any kind of confusion, let us now insist on the point that these functions concern the variation of the coefficients (102) with $R i$. They should be clearly distinguished from the usual damping functions used by some authors to characterise the damping of the total eddy viscosity and diffusivity, defined by

$$
\begin{aligned}
f_{\nu_{T}}(R i) & =\frac{\nu_{T}}{\nu_{T, 0}} \\
f_{K_{T}}(R i) & =\frac{K_{T}}{K_{T, 0}}
\end{aligned}
$$

where $\nu_{T, 0}=\nu_{T}(R i=0)$ and $K_{T, 0}=K_{T}(R i=0)$ (obviously, $\operatorname{Pr}_{T, 0}=$ $\left.\nu_{T, 0} / K_{T, 0}\right)$. The reason of this difference is that the formulae (101) are somewhat confusing by the fact that $k$ and $\varepsilon$ are also (implicitely) damped by buoyancy forces acting in (17) and (19). Hence, in principle $f_{\nu_{T}}<f_{C_{\mu}}$ and $f_{K_{T}}<$ $f_{C_{\nu}}$; however, for small $R i$ we may assume that $f_{\nu_{T}} \approx f_{C_{\mu}}$ and $f_{K_{T}} \approx f_{C_{\nu}}$. One also defines the turbulent Prandtl number damping function by

$$
f_{\operatorname{Pr}_{T}}(R i)=\frac{\operatorname{Pr}_{T}}{\operatorname{Pr}_{T, 0}}=\frac{f_{C_{\mu}}(R i)}{f_{C_{\nu}}(R i)}=\frac{f_{\nu_{T}}(R i)}{f_{K_{T}}(R i)}
$$

Finally, it is interesting to consider the flux Richardson number $R f$, defined by

$$
R f=-\frac{G_{k}}{P_{k}}=\frac{R i}{\operatorname{Pr}_{T}}
$$

The question of damping functions has been deeply investigated by a number of authors, and recent studies based on extensive analysis of experiments did not yield any firm conclusion ${ }^{25}$. Equations (92) and (93), together with Table 7 and values (105), yield theoretical expressions for $f_{C_{\mu}}(R i), f_{C_{\nu}}(R i)$ and $f_{\operatorname{Pr}_{T}}(R i)$. We may compare these to three other propositions available in the literature. We first consider the traditional experimental damping functions proposed by Munk and Anderson: ${ }^{1}$

$$
\begin{aligned}
f_{\nu_{T}}(R i) & =(1+10 R i)^{-1 / 2} \\
f_{K_{T}}(R i) & =\left(1+\frac{10}{3} R i\right)^{-3 / 2}
\end{aligned}
$$


This does not provide a complete set of data, since they do not give $\operatorname{Pr}_{T, 0}$. Thus we will use (110) together with the value of $\operatorname{Pr}_{T, 0}=0.75$ given by Mellor and Yamada ${ }^{2}$. As a second reference model, we consider Mellor and Yamada's, stating: ${ }^{2}$

$$
\begin{aligned}
f_{\nu_{T}}(R i) & =4.36 \frac{(0.213-R f)(0.269-R f)}{(1-R f)(0.25-R f)} \\
f_{K_{T}}(R i) & =4.69 \frac{0.213-R f}{1-R f} \\
R f & =0.725\left(R i+0.186-\sqrt{R i^{2}-0.316 R i+0.0346}\right)
\end{aligned}
$$

consistently with their value of $\operatorname{Pr}_{T, 0}$. Lastly, we will compare our model to Sukoriansky et al.'s, ${ }^{23}$ based on a theoretical spectral closure of stably stratified turbulence. These authors do not provide any explicit formulae; their results will be graphically plotted for comparison. In addition to these three models, we will also pay attention to two models for $\operatorname{Pr}_{T}$ only, firstly Ellison's model: ${ }^{26}$

$$
\operatorname{Pr}_{T}=\frac{(1-R f)^{2}}{1-R f / R f_{c}}
$$

with

$$
\begin{aligned}
R f & =\frac{2}{3}+\left(\sqrt{\frac{b^{2}}{4}+\frac{a^{3}}{27}}-\frac{b}{2}\right)^{1 / 3}-\left(\sqrt{\frac{b^{2}}{4}+\frac{a^{3}}{27}}+\frac{b}{2}\right)^{1 / 3} \\
a & =\frac{R i}{\operatorname{Pr}_{T, 0} R f_{c}}-\frac{1}{3} \\
b & =\left(\frac{2}{3 R f_{c}}-1\right) \frac{R i}{\operatorname{Pr}_{T, 0}}+\frac{2}{27}
\end{aligned}
$$

We will use this model with $R f_{c}=0.27$, according to Yamada, ${ }^{20}$ and the value of $\operatorname{Pr}_{T, 0}=0.75$ given by Mellor and Yamada. ${ }^{2}$ Secondly, we consider the model proposed by Zilitinkevich et al.: ${ }^{27}$

$$
\operatorname{Pr}_{T}=\frac{(1+19 R i)^{2.7}}{(1+36 R i)^{1.7}}
$$

with $\operatorname{Pr}_{T, 0}=0.80$ to estimate $R f$ from (109).

Figure 1 shows the damping functions and flux Richardson number versus gradient Richarsdon number for the present model (92) to (96) and the five reference models mentioned above. Generally speaking, all the models exhibit similar behaviour, although Munk and Anderson's predicts less damping than the others. As required, $f_{C_{\mu}}(R i)$ and $f_{C_{\nu}}(R i)$ are positive. The present model is very close to those of Mellor and Yamada for damping functions and to Zilitinkevich et al.'s for flux Richardson number. It is noticeable that the critical value of $R i$ does not affect the continuity of the turbulent Prandtl number, 
while $f_{C_{\mu}}(R i)$ and $f_{C_{\nu}}(R i)$ vanish at $R i=R i_{c}$. However, Sukorianski et al.'s model doesn't predict any turbulence destruction at large $R i$, contrary to our model and Mellor and Yamada's. On the contrary, $f_{C_{\mu}}(R i)$ tends to a constant while $f_{C_{\nu}}(R i)$ decreases gently to zero. This behaviour is also predicted by Zilitinkevich et al. As mentioned in the last section, it has been pointed out in many recent papers ${ }^{16}$ that under strong stratification, the vertical diffusion is not destructed. The reason is that at large $R i$, the density gradient tends to increase until a discontinuity appears, but this density interface is subject to oscillations (internal waves) which cause a flux of momentum (but still no scalar flux). In the next section, we will try to modify our model in order to take account of this phenomenon.

\subsection{Internal waves}

Zilitinkevich et al. ${ }^{27}$ suggest that the effect of internal waves could be accounted for by writting Rotta's coefficient $C_{R R}$ as a linear function of $R i$ in the return-toisotropy term $-C_{R R} \varepsilon \mathbf{a}$ of (22). However, this approach is subject to discussion since the action of internal waves is characterized by a time scale $1 / N$ rather than $\varepsilon / k$, where $N=S \sqrt{R i}$ is the Brünt-Väissälä frequency. Thus it would be more relevant to write $C_{R R}$ as a linear function of $\sqrt{R i}$, which leads to new problems in deriving an algebraic model. We propose here another method. At high $R i$, the presence of internal waves makes $f_{C_{\mu}}$ tending to a constant, as predicted by Sukoriansky et al. ${ }^{23}$ Thus, it turns as if $R i$ was a constant in the buoyancy term of the Reynolds stress equation (47). It is then possible to account for this effect by writting the model "constant" $C_{a 5}$ as a function of $R i$ :

$$
C_{a 5}(R i)=\frac{C_{a 6}}{1+C_{a 7} R i}
$$

$C_{a 6}$ having the value of $C_{a 5}$ in the model without internal waves. For small Richardson numbers, one recovers the original model $\left(C_{a 5}(R i)=C_{a 6}\right)$, while at high Richardson numbers the buoyancy term of Reynolds stresses, which reads $C_{a 5} R i$ in the final system (81), now tends to a constant $C_{a 6}^{\prime}$, as required:

$$
C_{a 5}(R i) R i \longrightarrow \frac{C_{a 6}}{C_{a 7}}=C_{a 6}^{\prime}
$$

Under the same condition (large $R i$ ), some of the model coefficients are modified:

$$
\begin{aligned}
R^{\prime \prime} & \longrightarrow-3 R \\
A_{\alpha 1} & \longrightarrow A_{\alpha 1, \infty}=-6 C_{a 3} C_{T 0} R=0.385 \\
A_{\xi 2} & \sim \frac{4 C_{a 6}^{\prime} C_{T 0}^{2}}{R i} \\
A_{D 1} & \longrightarrow 6 C_{a 2}^{2} C_{T 0} R \\
A_{D 3} & \sim \frac{6 C_{a 6}^{\prime} C_{T 0}^{2} R}{R i} \\
A_{D 4} & \longrightarrow 6 C_{T 0} R
\end{aligned}
$$


and

$$
\begin{aligned}
& B_{A 1} \quad \longrightarrow \quad B_{A 1, \infty}=-\frac{1}{3}\left(6 C_{T 0} R+A_{\xi 3}\right)=-0.579 \\
& B_{A 3} \quad \sim \quad B_{A 1, \infty}^{2}-\frac{8 C_{a 6}^{\prime} C_{T 0}^{2}(3 R+2)}{3 R i} \\
& B_{A 4} \quad \longrightarrow \quad B_{A 4, \infty}=2 B_{A 1, \infty} B_{A 2}-\frac{2}{3}\left(12 C_{a 2}^{2} C_{T 0} R-A_{\alpha 1, \infty}+2 A_{\xi 1}\right)=0.0177
\end{aligned}
$$

(here and in the following, $B_{A 1, \infty}$ is written for the limit of $B_{A 1}$ at large $R i$ $(R i \longrightarrow+\infty)$, etc. $)$. Thus:

$$
A \longrightarrow A_{\infty}=B_{A 2}+\frac{8 C_{a 6}^{\prime} C_{T 0}^{2}(3 R+2)-3 B_{A 4, \infty}}{6 B_{A 1, \infty}}
$$

and

$$
\begin{gathered}
\alpha \longrightarrow \alpha_{\infty}=\frac{A_{\alpha 1, \infty} A_{\infty}}{8 C_{a 6}^{\prime} C_{T 0}^{2}+A_{\xi 3} A_{\infty}+2 A_{\xi 1}-A_{\alpha 1, \infty}} \\
\xi \sim \frac{A_{\infty}\left(8 C_{a 6}^{\prime} C_{T 0}^{2}+A_{\xi 3} A_{\infty}+2 A_{\xi 1}\right)}{2\left(8 C_{a 6}^{\prime} C_{T 0}^{2}+A_{\xi 3} A_{\infty}+2 A_{\xi 1}-A_{\alpha 1, \infty}\right) R i}
\end{gathered}
$$

As expected, $\alpha$ tends to a constant (and so will do $f_{C_{\mu}}$, through (102) and (106)). It also appears that $\xi$ (and thus $f_{C_{\nu}}$ ) tends to zero as $R i^{-1}$. The value of $C_{a 6}^{\prime}$ can be chosen from the asymptotic value $f_{C_{\mu}, \infty}=0.228$ given by Sukoriansky et al., ${ }^{23}$ writing

$$
-\frac{\alpha_{\infty}}{2 C_{\mu, 0}}=f_{C_{\mu}, \infty}
$$

From (119) and (120), we obtain

$$
C_{a 6}^{\prime}=\frac{\left(\frac{B_{A 4, \infty}}{2 B_{A 1, \infty}}-B_{A 2}\right) B-2 A_{\xi 1}+A_{\alpha 1, \infty}}{4 C_{T 0}^{2}\left(2+\frac{3 R+2}{3 B_{A 1, \infty}} B\right)}
$$

where

$$
B=\frac{A_{\alpha 1, \infty}}{2 C_{\mu, 0} f_{C_{\mu}, \infty}}+A_{\xi 3}
$$

Numerically, we find $C_{a 6}^{\prime}=0.224$, and finally, from (116) and Table 6 :

$$
C_{a 7}=2.68
$$

Introducing (115) into our model (90) and (92) to (96) and re-arranging would lead to complicated formulae; thus we prefer keeping the previous set of formulae with a variable coefficient $C_{a 5}(R i)$ according to (115). The values of model constants from Table 6 must then be modified according to (94) to (96), 
leading to Table 8 . Figure 2 shows our modified model together with the models already presented in Figure 1. The expected behaviour at large $R i$ is now in a good agreement with Sukoriansky et al.'s predictions, particularly for $f_{C_{\nu}}$. We also show on the same figure experimental ${ }^{28,29,30,31,32,33,34,35}$ and numerical ${ }^{27,36}$

data. The agreement is generally satisfactory, particularly for $f_{C_{\nu}}$. Note that some of the data (experimental, ${ }^{28,32}$ but also numerical ${ }^{36}$ ) are in favour of the old-fashioned model of Munk and Anderson for the prediction of the turbulent Prandtl number and the flux Richardson number.

\subsection{Anisotropy and realizability}

The question of realizability is of fundamental importance for the theoretical prediction of turbulent fluxes. This complicated issue will be briefly treated here, focusing on Reynolds stresses. It has been known for a long time ${ }^{37}$ that the realizability of $\mathbf{R}$ is based on the second and third invariants of the anisotropy tensor a. With the general 2-D model (54), they read

$$
\begin{aligned}
& A_{2}=\operatorname{tr}\left(\mathbf{a}^{2}\right)=\left(\alpha^{2}+\beta^{2} \Omega^{* 2}+\gamma^{2} \frac{S^{* 2}}{12}\right) \frac{S^{* 2}}{2} \\
& A_{3}=\operatorname{tr}\left(\mathbf{a}^{3}\right)=\left(\alpha^{2}+\beta^{2} \Omega^{* 2}-\gamma^{2} \frac{S^{* 2}}{36}\right) \gamma \frac{S^{* 4}}{8}
\end{aligned}
$$

Alternatively, one can consider the quantities: ${ }^{6}$

$$
\begin{aligned}
\eta_{a} & =\left(\frac{A_{2}}{24}\right)^{1 / 2} \\
\xi_{a} & =\left(\frac{A_{3}}{48}\right)^{1 / 3}
\end{aligned}
$$

Then, realizability is achieved provided the following two inequalities are fulfilled:

$$
\left|\xi_{a}\right| \leq \eta_{a} \leq\left(\frac{1}{27}+2 \xi_{a}^{3}\right)^{1 / 2}
$$

or, identically:

$$
6 A_{3}^{2} \leq A_{2}^{3} \leq\left(\frac{8}{9}+A_{3}\right)^{3}
$$

From (125), it can be shown that the following inequality holds:

$$
A_{2}^{3}-6 A_{3}^{2}=\left(\alpha^{2}+\beta^{2} \Omega^{* 2}\right)\left(\alpha^{2}+\beta^{2} \Omega^{* 2}-\gamma^{2} \frac{S^{* 2}}{4}\right)^{2} \frac{S^{* 6}}{8} \geq 0
$$

Hence, the first of the two inequalities (128) is always satisfied. However, there is no evidence about the second one. With the present model (90) and (92) to $(96)$, with and without effect of internal waves, the evolution of $\eta_{a}$ versus $\xi_{a}$ in Lumley's triangle is presented on Figure 3 (top left) as a function of the gradient 
Richardson number (although the model without internal waves is less physical than the other one, we will still examine both models in this section). It appears that our models satisfies realizability except the model without internal waves when $R i$ reaches the critical value $R i_{c}$ given by (98), where the turbulence is supposed to be destroyed. Beyond this point, the realizability is broken because the model is supposed to predict a negative value of $S^{* 2}$, according to (97). The fact that at $R i=R i_{c}$ realizability fails from the first inequality of (128) is noticeable, because at this point the turbulence is almost isotropic instead of being two-dimensional. This means that for this model, while buoyancy affects turbulence, the ratio of vertical to horizontal velocity fluctuations remains finite. This state corresponds to an axisymmetric turbulence with a prolate Reynolds ellipsoid. On the other hand, internal waves keep $\xi_{a}$ (or, equivalently, $A_{3}$ ) almost constant with $R i$. However, one should keep in mind that the model with internal waves remains closer to the reality.

With the general assumption of simply stratified flows (section 4.2), the Reynolds stresses and scalar fluxes can be written in developed forms from (51) and (54) (with $S_{x x}^{*}=\Gamma_{T, x}^{*}=0$ ), to give

$$
\frac{\mathbf{R}}{k}=\left(\begin{array}{ccc}
\frac{2}{3}+\left(\frac{\gamma}{6}-\beta\right) \frac{S^{* 2}}{2} & 0 & \alpha \frac{S^{*}}{2} \\
0 & \frac{2}{3}-\frac{\gamma S^{* 2}}{6} & 0 \\
\alpha \frac{S^{*}}{2} & 0 & \frac{2}{3}+\left(\frac{\gamma}{6}+\beta\right) \frac{S^{* 2}}{2}
\end{array}\right)
$$

and

$$
\mathbf{Q}_{T}^{*}=\Gamma_{T}^{*}\left(\begin{array}{c}
\frac{1}{2} \zeta S^{*} \\
0 \\
\xi
\end{array}\right)
$$

Next, one defines the dimensionless mean fluctuation components $u^{+}, v^{+}$ and $w^{+}$along the $x, y$ and $z$ axis as

$$
u^{+}=\sqrt{\frac{R_{x x}}{k}}, \text { etc. }
$$

A relevant scalar measure of vertical anisotropy is then given by the ratio of vertical to horizontal kinetic energy:

$$
\sigma_{a}=\frac{w^{+2}}{u^{+2}+v^{+2}}=\frac{24}{16-(6 \beta+\gamma) S^{* 2}}-1
$$

With the assumption (91), it simplifies to

$$
\sigma_{a}=\frac{12 \alpha}{8 \alpha+6 \beta+\gamma}-1
$$

We may compare our model to Zilitinkevich et al. ${ }^{27}$ who suggest

$$
\sigma_{a}=\frac{1+c_{Z} R f}{4(1-R f)}\left[1-\left(1+\frac{1}{1+c_{Z} R f}\right) R f\right]
$$


with $c_{Z}=-2.25$. This formula must be used together with (109) and (114). Figure 3 (top right) shows $\sigma_{a}$ versus $R i$ with the present models (with and without internal waves) as well as (135) and experimental and numerical data. It appears that these data sets are two scattered to draw firm conclusions about the model quality. However, our models stand within the range of available data. As already mentioned by Zilitinkevich et al., we observe that $\sigma_{a}$ rapidly decreases in a narrow band of Richardson numbers around $R i_{c}$ (indicated on the figure, although it is no longer a critical value). At high $R i$ however, $\sigma_{a}$ remains finite. As previously mentioned, even the model without internal waves keeps a finite value of vertical-to-horizontal energy at high $R i$.

We finally examine the following two anisotropy parameters:

$$
\begin{aligned}
c_{a} & =-\frac{a_{x z}}{w^{+} \sqrt{u^{+2}+v^{+2}}} \\
c_{T} & =-\frac{Q_{T, z}^{*}}{w^{+} \sqrt{\theta^{*}}}
\end{aligned}
$$

which are always positive. Mauritsen and Svensson ${ }^{38}$ propose empirical dependency on $R i$ as

$$
\begin{aligned}
c_{a} & =\frac{0.278}{1+4 R i}+0.0925 \\
c_{T} & =\frac{0.3}{1+4 R i}
\end{aligned}
$$

Figure 3 (bottom) shows the functions $c_{a}(R i)$ and $c_{T}(R i)$ according to our model (with effect of internal waves) and (137). As $\sigma_{a}$ does, those two parameters decrease rapidly around $R i_{c}$, without any abrupt transition, to reach a constant value. According to the conclusions of Figure 2, this high- $R i$ limit is zero for $c_{T}$ (scalar anisotropy) while it is non-zero for $c_{a}$ (stress anisotropy). The present model globally shows the same shape as the empirical model, but give significantly different values of $c_{a}$ at large $R i$ and $c_{T}$ at small $R i$. However, it must be noticed that Mauritsen and Svensson's model (137) is based on observations showing large uncertainties. A careful attention at Figures 2 and 4 of Mauritsen and Svensson show that the predictions of our model stand within the range of their observations.

The values of anisotropy parameters at small and large $R i$ can be calculated from (99):

$$
\begin{aligned}
A_{2,0} & =-C_{a 3}=0.356 \\
A_{3,0} & =0 \\
\sigma_{a, 0} & =\frac{6}{4+3 C_{a 2}}-1=0.227 \\
c_{a, 0} & =3 \sqrt{\frac{2 C_{a 2}^{2}+C_{a 3}}{2\left(3 C_{a 2}-2\right)\left(3 C_{a 2}+4\right)}}=0.387 \\
c_{T, 0} & =\sqrt{\frac{C_{T 0}}{R}}=0.386
\end{aligned}
$$


The behaviour of these quantities at large $R i(R i \longrightarrow+\infty)$ can also be theoretically written from the model constants, leading to complicated formulae that we will not write here. Numerically, we obtain

$$
\begin{aligned}
\alpha_{\infty} & =-0.0413 \\
\beta_{\infty} & =-0.0122 \\
\gamma_{\infty} & \sim-\frac{0.00194}{R i} \\
\xi_{\infty} & \sim-\frac{0.00436}{R i}
\end{aligned}
$$

and

$$
\begin{aligned}
A_{2, \infty} & =0.333 \\
A_{3, \infty} & \sim-\frac{0.00989}{R i} \\
\sigma_{a, \infty} & =0.171 \\
c_{a, \infty} & =0.289 \\
c_{T, \infty} & \sim \frac{0.0821}{\sqrt{R i}}
\end{aligned}
$$

One may mention, for comparison, Zilitinkevich et al. ${ }^{27}$ who give $\sigma_{a, 0}=0.25$ and $\sigma_{a, \infty}=0.075$, according to Figure 3 .

\section{Conclusions}

An Explicit Algebraic Reynolds Stress and Scalar Flux Model (EARSSFM) has been presented with buoyancy effects through slight density variations, for simply-stratified turbulence (i.e. flows varying along the vertical direction). This model is gives algebraic formulae for the Reynolds stress tensor anisotropy a along with the active scalar flux vector $\mathbf{Q}_{T}^{*}$. It is summarized by equations (54), (57) and (92) to (96), together with (90), (115) and model constants given by Table 8 . The model proved to predict the damping of turbulent diffusion satisfactorily when compared to recent models and available exeprimental data. In particular, it predicts a correct value of the neutral turbulent Prandtl number (i.e. with no density stratification), and recovers the effect of internal waves at high Richardson numbers. The variation of turbulent Prandtl number versus gradient Richardson number $R i$ is also correctly predicted, as well as turbulence anisotropy. In comparaison to other existing models of theoretical or numerical damping functions, the present model presents the avantage of resulting directly from a consistent derivation of algebraic fluxes from governing equations. In particular, our model does not require any ad hoc constants.

One should temperate the conclusions of the above developments by making the following remark. All the Explicit Algebraic models available in the literature hold on the knowledge of local data, namely the mean velocity and 
scalar gradients $\mathbf{S}$ and $\boldsymbol{\Gamma}_{T}$ (resulting from the governing equations (1)), as well as the turbulent parameters $k, \varepsilon$ (obtained from equations (17) and (19), where the gradients play a prominent role through production and buoyancy). The performance of such a model in a numerical code is thus always dependent on the accuracy of the prediction of local gradients. Moreover, according to the comments made at the end of section 3.1, the equation (19) governing $\varepsilon$ is subject to many theoretical discussions. The ability of EARSSFM to provide good predictions is thus directly connected to the capabilities of the standard twoequation turbulence closure models and of the numerical schemes used to solve the latter. In this context, another question is the validity of the standard $k-\varepsilon$ model coefficients (Table 2) under variable $R i$.

Further work should consist of quantitative validations of the present model for passive and active scalars, based on DNS data of $\mathbf{S}, \boldsymbol{\Gamma}_{T}, k$ and $\varepsilon$ on a set of typical flows. The question of internal waves whould also be more deeply investigated from another viewpoint based on the dynamics of water waves.

\section{Acknowledgements}

The author tanks E. Toormann, from the Catholic University Leuven (Belgium), for fruitful discussions about the existence of a possible critical Richardson number. His ackowledgments also go to B. Galperin, from Princeton University (USA), S. Sukoriansky, from Ben-Gurion University of the Negev (Israel), and D. Stretch, from the University of KwaZulu-Natal (South Africa) for their data and comments, as well as to Martin Ferrand from Ecole des Ponts et Chaussées (France) for his careful review.

\section{Appendices: examples of algebraic decompo- sitions}

\subsection{Appendix 1: example of vector decomposition}

We give here an example of the decomposition of unknown vectors on the basis (56). Considering the case of the vector $\mathbf{S}^{*} \boldsymbol{\Omega}^{*} \boldsymbol{\Gamma}_{T}^{*}$, we use (62) to write

$$
\begin{aligned}
\mathbf{S}^{*} \boldsymbol{\Omega}_{T}^{*} \boldsymbol{\Gamma}_{T}^{*} & =\frac{\left(\mathbf{S}^{*} \boldsymbol{\Omega}^{*} \boldsymbol{\Gamma}_{T}^{*}\right) \cdot \boldsymbol{\Gamma}_{T}^{*}}{\left\|\mathbf{\Gamma}_{T}^{*}\right\|^{2}}+\frac{\left(\mathbf{S}^{*} \boldsymbol{\Omega}^{*} \boldsymbol{\Gamma}_{T}^{*}\right) \cdot\left(\boldsymbol{\Omega}^{*} \boldsymbol{\Gamma}_{T}^{*}\right)}{\left\|\boldsymbol{\Omega}^{*} \boldsymbol{\Gamma}_{T}^{*}\right\|^{2}} \mathbf{V}^{(2)} \\
& =\frac{1}{\Gamma_{T}^{* 2}}\left[\left(\boldsymbol{\Gamma}_{T}^{* T} \mathbf{S}^{*} \boldsymbol{\Omega}^{*} \boldsymbol{\Gamma}_{T}^{*}\right) \mathbf{V}^{(1)}+\frac{4}{\Omega^{* 2}}\left(\boldsymbol{\Gamma}_{T}^{* T} \boldsymbol{\Omega}^{*} \mathbf{S}^{*} \boldsymbol{\Omega}^{*} \boldsymbol{\Gamma}_{T}^{*}\right) \mathbf{V}^{(2)}\right]
\end{aligned}
$$

We used the relations $(\mathbf{A B}) \cdot \mathbf{C}=\mathbf{C}^{T} \mathbf{A B}$ for any tensor $\mathbf{A}$ and vectors $\mathbf{B}$ and $\mathbf{C}, \boldsymbol{\Omega}^{* T}=-\boldsymbol{\Omega}^{*}\left(\boldsymbol{\Omega}^{*}\right.$ is antisymmetric $)$, and $\left\|\boldsymbol{\Omega}^{*} \boldsymbol{\Gamma}_{T}^{*}\right\|=\Omega^{* 2}\left\|\boldsymbol{\Gamma}_{T}^{*}\right\| / 4\left(\boldsymbol{\Omega}^{*}\right.$ is 
a rotation tensor) together with (66). Then, we can write (in 2-D), from (51):

$$
\begin{aligned}
\boldsymbol{\Omega}^{*} \mathbf{S}^{*} \boldsymbol{\Omega}^{*} & =\Omega_{x z}^{* 2}\left(\begin{array}{ccc}
0 & 0 & 1 \\
0 & 0 & 0 \\
-1 & 0 & 0
\end{array}\right)\left(\begin{array}{ccc}
-S_{x z}^{*} & 0 & S_{x x}^{*} \\
0 & 0 & 0 \\
S_{x x}^{*} & 0 & S_{x z}^{*}
\end{array}\right) \\
& =\Omega_{x z}^{* 2}\left(\begin{array}{ccc}
S_{x x}^{*} & 0 & S_{x z}^{*} \\
0 & 0 & 0 \\
S_{x z}^{*} & 0 & -S_{x x}^{*}
\end{array}\right)=\frac{\Omega^{* 2}}{4} \mathbf{S}^{*}
\end{aligned}
$$

so that, immediately, with the definitions (68):

$$
\mathbf{S}^{*} \boldsymbol{\Omega}^{*} \boldsymbol{\Gamma}_{T}^{*}=S_{2} \mathbf{V}^{(1)}-S_{1} \mathbf{V}^{(2)}
$$

which is readily the second equation of (67).

\subsection{Appendix 2: example of a tensor decomposition}

Similarly to the algebraic calculations made in Appendix 1, we give an example of tensor decomposition on the basis (53). We first note that for any symmetric traceless tensor $\mathbf{B}$, the following identity holds:

$$
\left[\mathbf{A}^{S}-\frac{1}{3}(\operatorname{tr} \mathbf{A}) I\right]: \mathbf{B}=\mathbf{A}: \mathbf{B}
$$

(reminding that $\mathbf{A}^{S}$ is the symmetric part of the tensor $\mathbf{A}$ ). Next, as an example we consider the tensor $\mathbf{C}=\left(\mathbf{e}_{z} \otimes \mathbf{Q}_{T}^{*}\right)^{S}-\frac{1}{3}\left(\mathbf{e}_{z} \cdot \mathbf{Q}_{T}^{*}\right) \mathbf{I}$ appearing in (47). We first write the first component of $\mathbf{C}$ 's decomposition, according to (57), (63), (66) and (144), as

$$
\begin{aligned}
\frac{\mathbf{C}: \mathbf{T}^{(1)}}{\left\|\mathbf{T}^{(1)}\right\|^{2}} & =\frac{\left(\mathbf{e}_{z} \otimes \mathbf{Q}_{T}^{*}\right): \mathbf{S}^{*}}{\left\|\mathbf{S}^{*}\right\|^{2}} \\
& =\frac{2}{S^{* 2}}\left(\xi \mathbf{e}_{z} \otimes \boldsymbol{\Gamma}_{T}^{*}+\zeta \mathbf{e}_{z} \otimes \boldsymbol{\Omega}^{*} \boldsymbol{\Gamma}_{T}^{*}\right): \mathbf{S}^{*} \\
& =\frac{2}{S^{* 2}}\left(\xi \mathbf{e}_{z}^{T} \mathbf{S}^{*} \boldsymbol{\Gamma}_{T}^{*}+\zeta \mathbf{e}_{z}^{T} \mathbf{S}^{*} \boldsymbol{\Omega}^{*} \boldsymbol{\Gamma}_{T}^{*}\right)
\end{aligned}
$$

We used the relation $(\mathbf{A} \otimes \mathbf{B}): \mathbf{C}=\mathbf{A}^{T} \mathbf{C B}$ for any tensor $\mathbf{C}$ and vectors $\mathbf{A}$ and $\mathbf{B}$. Next, from (56) and (67) we can write

$$
\begin{aligned}
\frac{\mathbf{C}: \mathbf{T}^{(1)}}{\left\|\mathbf{T}^{(1)}\right\|^{2}} & =\frac{2}{S^{* 2}}\left(\xi S_{1} \mathbf{e}_{z} \cdot \mathbf{V}^{(1)}+\xi \frac{4 S_{2}}{\Omega^{* 2}} \mathbf{e}_{z} \cdot \mathbf{V}^{(2)}+\zeta S_{2} \mathbf{e}_{z} \cdot \mathbf{V}^{(1)}-\zeta S_{1} \mathbf{e}_{z} \cdot \mathbf{V}\left({ }^{(2} 4\right)\right. \\
& =\frac{2}{S^{* 2}}\left[\xi\left(S_{1} \Gamma_{1}+\frac{4}{\Omega^{* 2}} S_{2} \Gamma_{2}\right)+\zeta\left(S_{2} \Gamma_{1}-S_{1} \Gamma_{2}\right)\right]
\end{aligned}
$$

The two other components of $\mathbf{C}$ along the axis corresponding to the tensors $\mathbf{T}^{(2)}$ and $\mathbf{T}^{(3)}$ are calculated similarly. In the subsequent algebra required to 
treat the second (tensorial) equation of the system (47), we use the following two identities:

$$
\begin{aligned}
S_{1}^{2}+\frac{4}{\Omega^{* 2}} S_{2}^{2} & =\frac{S^{* 2}}{4} \\
\Gamma_{1}^{2}+\frac{4}{\Omega^{* 2}} \Gamma_{2}^{2} & =\Gamma_{T}^{* 2}
\end{aligned}
$$

which simply result from the orthogonality of the basis $\left\{\mathbf{T}^{(i)}\right\}$ and $\left\{\mathbf{V}^{(i)}\right\}$.

\section{References}

${ }^{1}$ W. H. Munk and E. A. Anderson, "Notes on a theory of the thermocline," J. Marine Research 3, 276 (1948).

${ }^{2}$ G. L. Mellor and T. Yamada, "A hierarchy of turbulence closure models for planetary boundary layers," J. Atmosph. Sciences 31, 1792 (1974).

${ }^{3}$ B. Galperin, S. Sukoriansky and P. S. Anderson, "On the critical Richardson number in stably stratified turbulence," Atmosph. Sc. Letters 8, 65 (2007).

${ }^{4}$ S. B. Pope, "A more general effective-viscosity hypothesis," J. Fluid Mech. 72, 331 (1975).

${ }^{5}$ P. M. Wikström, S. Wallin and A. V. Johansson, "Derivation and investigation of a new explicit algebraic model of the passive scalar flux," Phys. Fluids 12, 688 (2000).

${ }^{6}$ S. B. Pope, Turbulent Flows (Cambridge University Press, Cambridge, 2000).

${ }^{7}$ B. E. Launder, G. J. Reece and W. Rodi, "Progress in the development of a Reynolds-stress turbulence closure," J. Fluid Mech. 68, 537 (1975).

8 N. Wallin and A. V. Johansson, "An explicit algebraic Reynolds stress model for incompressible and compressible turbulent flows," J. Fluid Mech. 403, 89 (2000).

${ }^{9}$ W. Rodi, Turbulence Models and Their Applications in Hydraulics (IAHR monograph, Brookfield, Rotterdam, 2000).

10 V. Yakhot and L. M. Smith, "The renormalization group, the epsilonexpansion and derivation of turbulence models," Journ. Sc. Comput. 7, 35 (1992).

${ }^{11}$ B. E. Launder and D. B. Spalding, Mathematical Models of Turbulence (Academic Press, London, 1972).

12 C. M. Högström, S. Wallin and A. V. Johansson, "Passive scalar flux modelling for CFD," in $2^{\text {nd }}$ International Symposium on Turbulence and Shear Flow Phenomena, (Stockholm, 2001), Vol. II, p. 383.

${ }^{13}$ F. Schwertfirm and M. Manhart, "DNS of passive scalar transport in turbulent channel flow at high Schmidt numbers," Int. J. Heat and Fluid Flow 28, 1204 (2007).

14 M. M. Gibson and B. E. Launder, "On the calculation of horizontal, turbulent free shear flow under gravitational influence," J. Heat Transfer, Trans. ASME 98, 81 (1976). 
15 H. Hattori, A., Morita and Y. Nagano, "Nonlinear eddy diffusivity models reflecting buoyancy effect for wall-shear flow and heat transfer," Int. J. Heat and Fluid Flow 27, 671 (2006).

16 B. Galperin, L. H. Kantha, S. Hassid and A. Rosati, " A quasi-equilibrium turbulent energy model for geophysical flows," J. Atmosph. Sciences 45, 55 (1988).

17 T. B. Gatski and C. G. Speziale, "On explicit algebraic stress models for complex turbulent flows," J. Fluid Mech. 254, 59 (1993).

18 S. S. Girimaji, "Fully-explicit and self-consistent algebraic Reynolds-stress model," report No. 95-82, ICASE, 1995.

19 J. W. Miles and L. N. Howard, "Note on a heterogeneous shear flow," J. Fluid Mech. 20, 311 (1964).

20 T. Yamada, "The critical Richardson number and the ratio of the eddy transport coefficients obtained from a turbulence closure model," J. Atmosph. Sciences 32, 926 (1975).

21 G. L. Mellor and T. Yamada, "Development of a turbulence closure model for geophysical fluid problems," Reviews of Geophysics ans Space Physics 20, 851 (1982).

22 J. S. Turner, Buoyancy Effects in Fluids (Cambridge University Press, Cambridge, 1973).

23 S. Sukoriansky, B. Galperin and V. Perov, "Application of a new spectral theory of stably stratified turbulence to the atmospheric boundary layer over sea ice," Boundary-Layer Meteorology 117, 231 (2005).

24 J.-C. Galland, "Transport de sédiments en suspension et turbulence," report No. HE42/96/007/A, EDF R\&D (1996, in French).

25 E. A. Toorman, A. W. Bruens, C. Kranenburg and J. C. Winterwerp, "Interaction of suspended cohesive sediment and turbulence," in Fine Sediment Dynamics in the Marine Environment (Elsevier publications, Amsterdam, 2002), p. 7.

26 T. H. Ellison, "Turbulent transport of heat and momentum from an infinite rough plane," J. Fluid Mech. 2, 456 (1957).

27 S. S. Zilitinkevich, T. Elperin, N. Kleeorin and I. Rogachevskii, "Energyand flux-budget (EFB) turbulence closure model for the stably stratified flows. Part I: Steady-state, homogeneous regimes," Boundary-Layer Meteorol. 125, 167 (2007).

28 Y. Ohya, "Wind-tunnel study of atmospheric stable boundary layers over a rough surface," Boundary-Layer Meteorology 98, 57 (2001).

29 L. Mahrt and D. Vickers, "Boundary layer adjustment over small-scale changes of surface heat flux," Boundary-Layer Meteorol. 116, 313 (2005).

30 G. S. Poulos, W. Blumen, D. C. Fritts, J. K. Lundquist, J. Sun, S. P. Burns, C. Nappo, R. Banta, R. Newsom, J. Cuxart, E. Terradellas, B. Balsley and M. Jensen, "CASES-99: a comprehensive investigation of the stable nocturnal boundary layer," Bull. Amer. Meteorol. Soc. 83, 555 (2002).

31 T. Uttal, J. A. Curry, M. G. McPhee, D. K. Perovitch and 24 other coauthors, "Surface heat budget of the Arctic Ocean," Bull. American Meteorol. Soc. 83, 255 (2002). 
32 E. J. Strang and H. J. S. Fernando, "Vertical mixing and transports through a stratified shear layer," J. Phys. Oceanogr. 31, 2026 (2001).

${ }^{33}$ C. R. Rehmann and J. R. Koseff, "Mean potential energy change in stratified grid turbulence," Dynamics of Atmospheres and Oceans 37, 271 (2004).

${ }^{34}$ F. Bertin, J., Barat and R. Wilson, "Energy dissipation rates, eddy diffusivity, and the Prandtl number: an in situ experimental approach and its consequences on radar estrimate of turbulence parameters," Radio Sciences $\mathbf{3 2}$, 791 (1997).

35 J. Kondo, O. Kanechika and N. Yasuda, "Heat and momentum transfer under strong stability in the atmospheric surface layer," J. Atmosph. Sciences 35, 1012 (1978).

${ }^{36}$ D. D. Stretch, J. W. Rottman, S. K. Venayagamoorthy, K. K. Nomura and C. R. Rehmann, "Mixing efficiency in decaying stably stratified turbulence," Dynamics of Atmospheres and Oceans (in press), 10.1016/j.dynatmoce.2008.11.002.

37 J. L. Lumley, "Computational modeling of turbulent flows," Adv. Applied Mech. 18, 123 (1977).

38 T. Mauritsen and G. Svensson, "Observations of stably stratified sheardriven atmospheric turbulence at low and high Richardson numbers," J. Atmosph. Sciences 64, 645 (2007).

\begin{tabular}{c|cccccc}
\hline Model & $C$ & $C_{R R}$ & $C_{R P}$ & $C_{R Q}$ & $C_{R G}$ & $C_{R S}$ \\
\hline \hline LRR & 0.4 & 1.5 & 0.76 & 0.11 & 0.55 & 0.36 \\
\hline WJ & $\frac{5}{9}$ & 1.8 & 0.78 & 0.22 & 0.55 & 0.53
\end{tabular}

Table 1 - Model constants for the LRR-QI model, according to Launder, Reece and Rodi $(\mathrm{LRR})^{7}$ and Wallin and Johansson (WJ) ${ }^{8}$.

\begin{tabular}{ccc}
\hline$C_{\varepsilon 1}$ & $C_{\varepsilon 2}$ & $C_{\varepsilon 3}$ \\
\hline \hline 1.44 & 1.92 & 1.0 \\
\hline
\end{tabular}

Table 2 - Model constants ${ }^{11}$ of the equation governing $\varepsilon$.

\begin{tabular}{c|ccccc}
\hline Model & $B_{a 1}$ & $B_{a 2}$ & $B_{a 3}$ & $B_{a 4}$ & $B_{a 5}$ \\
\hline \hline LRR & 0.13 & 0.35 & $\frac{8}{15}$ & 0.085 & 0.90 \\
\hline WJ & 0 & 0.44 & $\frac{8}{15}$ & 0 & 0.90 \\
\hline
\end{tabular}

Table 3 - Model constants for the final Reynolds stress model (22).

\begin{tabular}{c|ccccc}
\hline Model & $C_{T Q}$ & $C_{T \Gamma}$ & $C_{T \Gamma}^{\prime}$ & $C_{T R}$ & $C_{T G}$ \\
\hline \hline HWJ & 4.51 & -0.47 & 0.02 & 0.08 & 0.5 \\
\hline WWJ & $1.6\left(1+\frac{\tau}{\tau_{\theta}}\right)$ & 0 & 0 & 0 & 0.5 \\
\hline
\end{tabular}

Table 4 - Model constants for pressure-scalar gradient correlation model, according to Högström et al. (HWJ) ${ }^{12}$ and Wikström et al. (WWJ). ${ }^{5}$

\begin{tabular}{c|cccc}
\hline Model & $B_{T 1}$ & $B_{T 2}$ & $B_{T 3}$ & $B_{T 4}$ \\
\hline \hline HWJ & 2.90 & 2.98 & 1.84 & 1.0 \\
\hline WWJ & 2 & 2 & 2 & 1.0 \\
\hline
\end{tabular}


Table 5 - Model constants of the final scalar flux model (37).

\begin{tabular}{ccccc}
\hline$C_{a 2}$ & $C_{a 3}$ & $C_{a 5}$ & $C_{T 0}$ & $R^{\prime \prime}$ \\
\hline \hline 0.296 & -0.356 & 0.6 & 0.164 & -2.1
\end{tabular}

Table 6 - Model constants with assumption (88).

\begin{tabular}{ccccccccc}
\hline$A_{\alpha 1}$ & $A_{\alpha 2}$ & $A_{\xi 1}$ & $A_{\xi 2}$ & $A_{\xi 3}$ & $B_{A 1}$ & $B_{A 2}$ & $B_{A 3}$ & $B_{A 4}$ \\
\hline \hline 0.280 & 1.07 & 0.00563 & 0.0646 & 0.656 & -0.809 & 0.0907 & 0.375 & -0.0673
\end{tabular}

Table 7 - Constants of the model (92) to (96).

\begin{tabular}{cccccc}
\hline$C_{a 2}$ & $C_{a 3}$ & $C_{a 6}$ & $C_{a 7}$ & $C_{T 0}$ & $R$ \\
\hline \hline 0.296 & -0.356 & 0.6 & 2.68 & 0.164 & 1.1 \\
\hline
\end{tabular}

Table 8 - Model constants of the final model with internal waves, given by equations (90), (92) to (96) and (115).
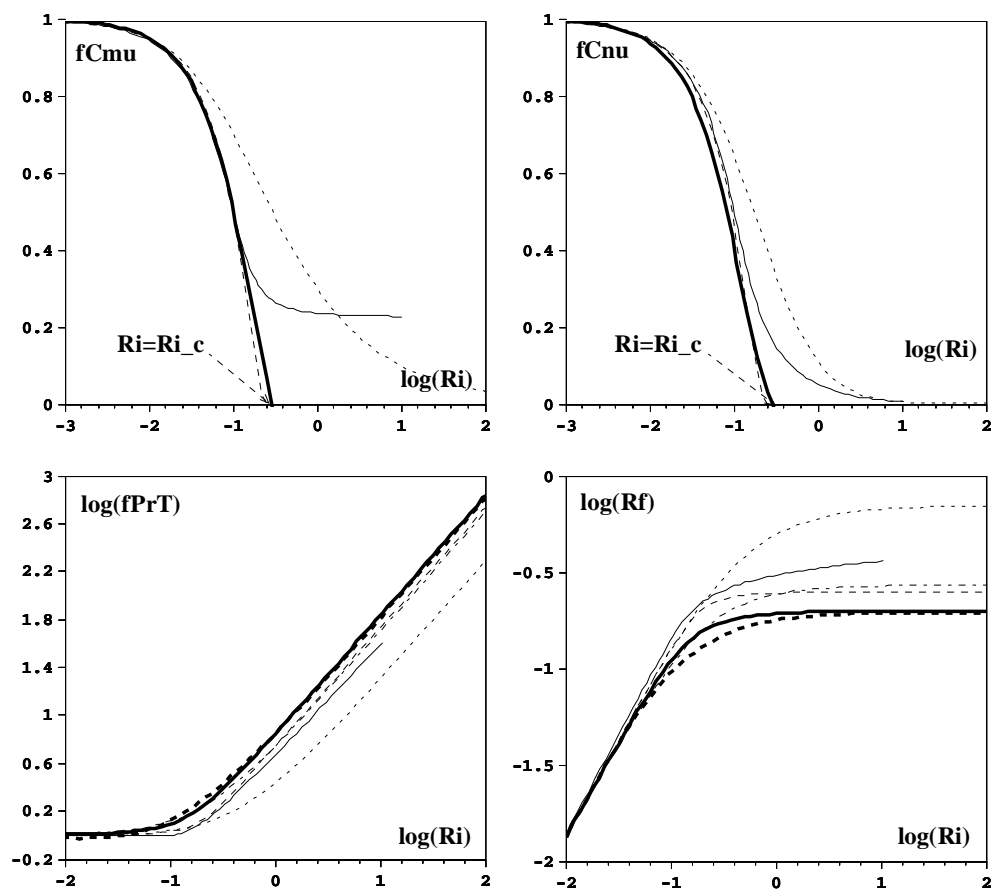
Figure 1: Damping functions $f_{C_{\mu}}(R i)$ (top left), $f_{C_{\nu}}(R i)$ (top right) and $f_{\mathrm{Pr}_{t}}(R i)$ (bottom left) and flux Richardson number $R f$ (bottom right) versus gradient Richardson number Ri. Bold solid line: present model without accounting for internal waves; solid thin line: Sukoriansky et al. $;^{23}$ dashed line: Mellor and Yamada; ${ }^{2}$ dotted line: Munk and Anderson $;{ }^{1}$ bold dashed line: Zilitinkevich et al. ${ }^{27}$
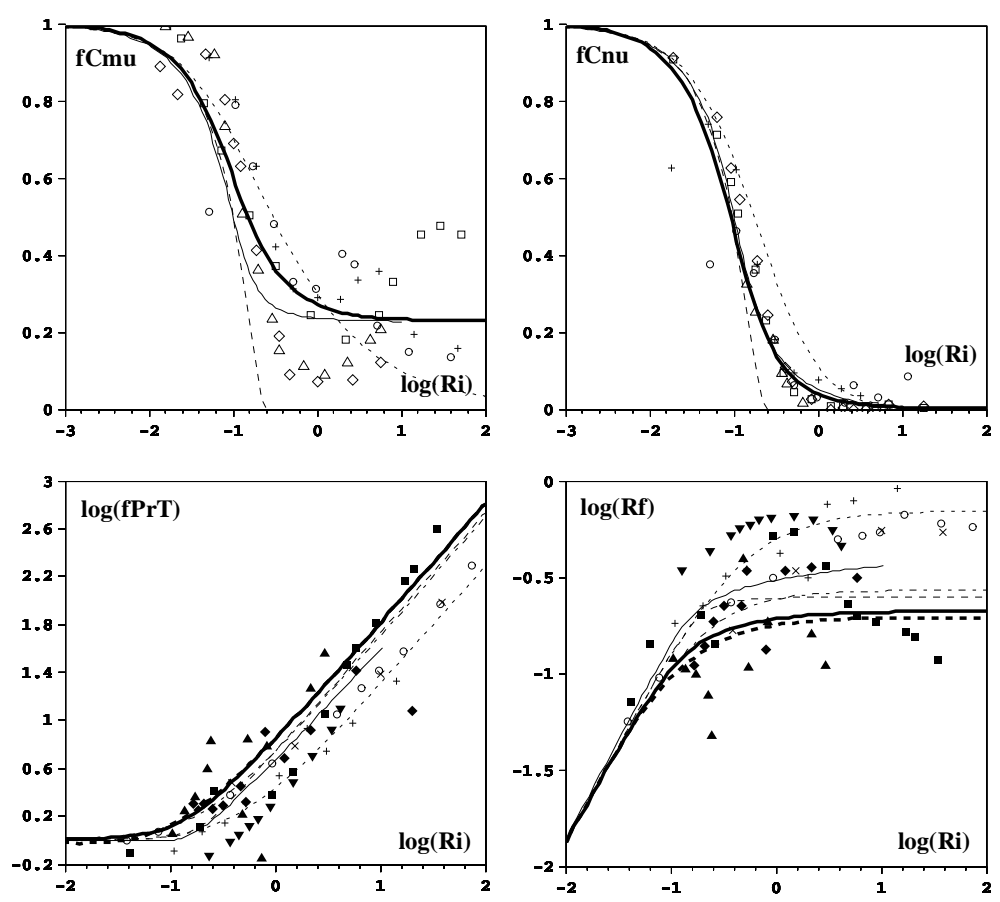

Figure 2: Same as Figure 1, the present model (bold solid line) accounting now for internal waves. The symbols are experimental and numerical data: empty circles: Ohya $;^{28}$ empty squares: Mahrt and Vickers; ${ }^{29}$ empty diamonds: Poulos et al. $;^{30}$ empty triangles: Uttal et al. $;^{31}$ black overturned triangles: Strang and Fernando; ${ }^{32}$ black squares: Rehmann and Koseff; ${ }^{33}$ black triangles: Bertin et al. ${ }^{34}$ black diamonds: Kondo et al. ${ }^{35}$ crosses: LES by Zilitinkevitch et al. ${ }^{27}$ slanting crosses: DNS by Stretch et al. ${ }^{36}$ 



Figure 3: Top left: the Lumley triangle $\left(\xi_{a}, \eta_{a}\right)$ (the rectangle is a zoom); top right: $\sigma_{a}$ versus $R i$; bottom left: $c_{a}$ versus $R i$; bottom right: $c_{T}$ versus $R i$. Solid lines: present model with internal waves; dashed lines: present model without internal waves; dotted line: Zilitinkevich et al. $;^{27}$ dashed-dotted line: Mauritsen and Svensson. ${ }^{38}$ The symbols are experimental and numerical data, with nomenclature identical as in Figure 2. 